\title{
Redshifted X-rays from the material accreting onto TW Hydrae: Evidence of a low-latitude accretion spot
}

\author{
C. Argiroffi ${ }^{1,2}$, J. J. Drake ${ }^{3}$, R. Bonito ${ }^{2}$, S. Orlando ${ }^{2}$, G. Peres ${ }^{1,2}$, and M. Miceli ${ }^{1,2}$ \\ 1 Dip. di Fisica e Chimica, Università di Palermo, Piazza del Parlamento 1, 90134 Palermo, Italy \\ e-mail: argi@astropa.unipa.it \\ 2 INAF - Osservatorio Astronomico di Palermo, Piazza del Parlamento 1, 90134 Palermo, Italy \\ 3 Smithsonian Astrophysical Observatory, MS-3, 60 Garden Street, Cambridge, MA 02138, USA
}

Received 9 June 2017 / Accepted 8 August 2017

\begin{abstract}
Context. High resolution spectroscopy, providing constraints on plasma motions and temperatures, is a powerful means to investigate the structure of accretion streams in classical T Tauri stars (CTTS). In particular, the accretion shock region, where the accreting material is heated to temperatures of a few million degrees as it continues its inward bulk motion, can be probed by X-ray spectroscopy. Aims. In an attempt to detect for the first time the motion of this X-ray-emitting post-shock material, we searched for a Doppler shift in the deep Chandra High Energy Transmission Grating observation of the CTTS TW Hya. This test should unveil the nature of this $\mathrm{X}$-ray emitting plasma component in CTTS and constrain the accretion stream geometry.

Methods. We searched for a Doppler shift in the X-ray emission from TW Hya with two different methods: by measuring the position of a selected sample of emission lines and by fitting the whole TW Hya X-ray spectrum, allowing the line-of-sight velocity to vary. Results. We found that the plasma at $T \sim 2-4 \mathrm{MK}$ has a line-of-sight velocity of $38.3 \pm 5.1 \mathrm{~km} \mathrm{~s}^{-1}$ with respect to the stellar photosphere. This result definitively confirms that this X-ray-emitting material originates in the post-shock region, at the base of the accretion stream, and not in coronal structures. The comparison of the observed velocity along the line of sight, $38.3 \pm 5.1 \mathrm{~km} \mathrm{~s}^{-1}$, with the inferred intrinsic velocity of the post shock of TW Hya, $v_{\text {post }} \approx 110-120 \mathrm{~km} \mathrm{~s}^{-1}$, indicates that the footpoints of the accretion streams on TW Hya are located at low latitudes on the stellar surface.

Conclusions. Our results indicate that complex magnetic field geometries, such as those of TW Hya, permit low-latitude accretion spots. Moreover, since on TW Hya the redshift of the soft X-ray emission is very similar to that of the narrow component of the C IV resonance doublet at $1550 \AA$, then the plasma at 2-4 MK and that at 0.1 MK likely originate in the same post-shock regions.
\end{abstract}

Key words. accretion, accretion disks - stars: pre-main sequence - stars: variables: T Tauri, Herbig Ae/Be - X-rays: stars techniques: spectroscopic

\section{Introduction}

Low-mass stars $\left(M \lesssim 2 M_{\odot}\right)$, at the initial stages of their premain-sequence evolution, are surrounded by circumstellar disks from which they accrete material. During this phase they are classified as classical T Tauri stars (CTTS). The accretion phase persists for a few Myr, during which the circumstellar disk gradually exhausts its gas and dust content through accretion itself, photoevaporation, and planet formation (Armitage 2011; Williams \& Cieza 2011). During the accretion phase, the star exchanges mass, angular momentum, and energy with its surrounding environment. These exchanges are thought to affect the stellar evolution, in terms of rotation, internal structure, bolometric luminosity, and lithium abundance, well beyond the accretion phase itself (Bouvier et al. 1997; Baraffe et al. 2009; Baraffe \& Chabrier 2010).

Both observational and theoretical evidence clearly indicates that the magnetic field plays a major role in the accretion process of CTTS (see Romanova \& Owocki 2015; Hartmann et al. 2016, and references therein). The so-called magnetospheric accretion model predicts that the stellar magnetic field disrupts the inner disk, loads disk material in its flux tubes, and guides this material in its fall toward the star (e.g., Königl 1991; Calvet \& Hartmann 1992; Hartmann et al. 1994). Because of the typical gravitational wells of CTTS, the accreting material accelerates up to
$300-500 \mathrm{~km} \mathrm{~s}^{-1}$ and terminates its fall impacting onto the denser stellar atmosphere. There, at the footpoints of these accretion streams, strong shocks convert the bulk of the kinetic energy of the accreting material into thermal energy (Calvet \& Gullbring 1998) and generate hot spots on the stellar surface. Because of the high pre-shock velocities, the post-shock material, located just behind the shock surface, is expected to have temperatures of a few MK, and hence to radiate mainly in the X-ray band (Ulrich 1976; Königl 1991; Gullbring 1994). The observed emission of these accretion spots peaks instead in the UV and optical bands, indicating temperatures of $\sim 10^{4} \mathrm{~K}$ (Ingleby et al. 2013). These temperatures, cooler than that expected, are thought to be related to a significant reprocessing of the primary X-rays into longer wavelength photons in the accretion-shock region itself. In fact, local X-ray absorption due to the surrounding stellar atmosphere and pre-shock material, and their consequent heating, could be substantial (Drake 2005; Sacco et al. 2010; Bonito et al. 2014; Costa et al. 2017).

However, despite this expected local absorption, recent findings hint that some of the primary X-ray photons are in any case able to escape from the accretion-shock region. High resolution $\mathrm{X}$-ray spectroscopy $(R \sim 1000)$ of CTTS in fact proved the existence of a cool $(T \sim 3 \mathrm{MK})$ and high-density $\left(n_{\mathrm{e}} \sim 10^{12} \mathrm{~cm}^{-3}\right)$ plasma component (e.g., Kastner et al. 2002; Stelzer \& Schmitt 2004; Schmitt et al. 2005; Günther et al. 2006), as well as 
high-temperature and low-density coronal plasmas typical for low-mass stars. Several hydrodynamic (HD) and magnetohydrodynamic (MHD) models investigated the physical conditions of the accretion-shock region (e.g., Sacco et al. 2008, 2010; Orlando et al. 2010, 2013; Matsakos et al. 2013), indicating that this high-density plasma at a few MK, which was never observed in non-accreting low-mass stars, is exactly what would be produced in an accretion shock on a CTTS. Recently, indications of X-rays emitted in accretion shocks were also obtained with low resolution X-ray spectroscopy $(R \sim$ 40 ), thanks to simultaneous optical monitoring (Guarcello et al. 2017). All these findings make soft X-rays one of the most direct probes of the accretion-shock region. The diagnostic power offered by soft X-rays from CTTS is illustrated by their power to infer the densities and velocities of the stream (e.g., Kastner et al. 2002; Stelzer \& Schmitt 2004; Günther et al. 2006; Argiroffi et al. 2007), the peculiar chemical composition of the inner disk material (Drake et al. 2005), indications on the accretion stream geometry (Argiroffi et al. 2011, 2012), and the intrinsic variability of the accretion stream properties (Brickhouse et al. 2012).

Beside several theoretical and observational lines of evidence supporting the hypothesis that soft X-rays in CTTS come from plasma heated in the accretion shock, other results appear to conflict with this scenario: the lack of correlation between the soft X-ray excess with UV accretion indicators (Güdel \& Telleschi 2007), the lack of a high-density plasma component on the CTTS T Tauri (Güdel et al. 2007), and the density versus temperature pattern of X-ray emitting plasma (Brickhouse et al. 2010). Starting from these findings, alternative scenarios were proposed, suggesting that the cool plasma in CTTS could also be composed of coronal structures modified, cooled, or fed by accretion (Güdel \& Telleschi 2007; Brickhouse et al. 2010; Schneider et al. 2017).

While the most important mechanisms governing magnetospheric accretion are understood, the geometry of the magnetosphere is still poorly constrained. Modeling of accretion onto CTTS usually assumes that disk disruption and material loading is due to the dipole component of the magnetic field (e.g., Hartmann et al. 1994; Romanova et al. 2003, 2011) because this component is expected to be dominant at large distance. However, the geometry of the accretion streams, and consequently their footpoint locations, depends strongly on the magnetic configuration near the stellar surface. A dipole component that also dominates near the stellar surface would anchor the accretion stream footpoints at high latitudes (Romanova et al. 2004). Conversely, surface magnetograms indicate that CTTS have complex magnetic field configurations that are often characterized by intense higher order components (e.g., Gregory et al. 2008; Donati \& Landstreet 2009). Higher order components could instead generate accretion streams terminating at low latitude on the stellar surface (Gregory et al. 2006).

In addition to the magnetic field, accretion geometry could also be related to the inner structure of circumstellar disks. At ages of a few Myr, circumstellar disks often show complex and asymmetrical structures, such as inner disk warps and misalignments (e.g., Hashimoto et al. 2011; Apai et al. 2015; Debes et al. 2017), which are possibly due to ongoing planet formation. These asymmetries, if they extend to the inner disk rim, could affect the accretion stream geometry.

To provide constraints on the accretion geometry, we test in this work one basic prediction of accretion-related X-rays that has not been investigated yet. The velocity jump predicted for strong-shock conditions across the shock surface, at the base of the accretion stream, is $v_{\text {post }}=v_{\text {pre }} / 4$ (Zel'dovich \& Raizer $1967)$, where $v_{\text {post }}$ and $v_{\text {pre }}$ indicate the post- and pre-shock velocities, respectively. Therefore the plasma located just behind the shock surface is expected to have a downward bulk velocity $v_{\text {post }}$ of $\sim 100 \mathrm{~km} \mathrm{~s}^{-1}$ within the stellar atmosphere ${ }^{1}$. The emerging X-ray spectrum should therefore be redshifted with respect to the stellar photosphere. Hydrodynamic and MHD simulations have also confirmed this. In fact, even if the post-shock region is characterized by oscillations due to the classical radiative shock instability that is not detected (e.g., Drake et al. 2009), the bulk post-shock velocity is still expected to vary between $\sim v_{\text {pre }} / 8$ and $\sim v_{\text {pre }} / 2$ (Sacco et al. 2008, 2010). Performing temporal and spatial integration of the shock region, and considering the local absorption, the expected redshift should still be detectable with the present-day X-ray spectrometers, depending on the viewing angle under which the shock region is observed (Bonito et al. 2014; Bonito et al., in prep.).

Our aim is therefore to search for a Doppler shift in the X-ray emission from CTTS to measure the bulk velocity of the plasma components at a few MK with respect to the stellar photosphere. First of all, the detection of a redshift would be definitive evidence that the high-density plasma in CTTS is indeed located in the post-shock region and not in accretion-related coronal structures. The detected velocity could also provide constraints on the inclination angle under which the shock region is observed, and hence on the geometry of the accretion. Finally, the comparison of any detected velocity of the X-emitting plasma with that obtained from far UV lines also ascribed to the shock region (e.g., Ardila et al. 2013), also allows us to construct a coherent picture of the accretion-shock region in CTTS. To this aim, we selected the nearest CTTS, TW Hya, because its almost pole-on orientation minimizes rotational modulation effects and maximizes the possibility to observe redshifts in case of high-latitude accretion shocks. Moreover, also because of its proximity and hence its high X-ray flux, TW Hya was targeted for a Large Observing Project with the Chandra High Energy Transmission Gratings Spectrometer (HETGS), the instrument that currently provides the highest spectral resolution in the soft X-ray band.

We summarize the main properties of TW Hya in Sect. 2. In Sect. 3 we scrutinize the expected resolution of the Chandra/HETGS, and in Sect. 3 we detail the analyzed data sets. In Sect. 4, we present the two methods adopted to measure X-ray Doppler shifts, reporting the obtained results in Sect. 5, and discussing these results in Sect. 6.

\section{TW Hya}

Located at $59.5 \mathrm{pc}$ (Gaia Collaboration 2016), TW Hya is an $8 \mathrm{Myr}$ old CTTS and the eponymous member of the TW Hya Association (Kastner et al. 1997; Webb et al. 1999; Donaldson et al. 2016). The spectral type classification of TW Hya is uncertain, ranging from K7 to M2.5 depending on the wavelength band in which the classification is made (see Vacca \& Sandell 2011; Debes et al. 2013; Herczeg \& Hillenbrand 2014, and references therein). As a consequence the mass and radius estimates of TW Hya are also uncertain, with $M_{\star}$ ranging from $\sim 0.5$ to $\sim 0.8 M_{\odot}$ and $R_{\star}$ ranging from $\sim 0.8$ to $\sim 1.1 R_{\odot}$ (Donati et al. 2011; Debes et al. 2013). TW Hya is surrounded by a circumstellar disk, with

1 This expected value for $v_{\text {post }}$ is based on several observational and theoretical arguments; the most stringent argument is the fact that $v_{\text {pre }}$ of at least $300 \mathrm{~km} \mathrm{~s}^{-1}$ (corresponding to $v_{\text {post }} \approx 75 \mathrm{~km} \mathrm{~s}^{-1}$ ) are needed to have X-ray emission from the post-shock plasma (Sacco et al. 2010). 


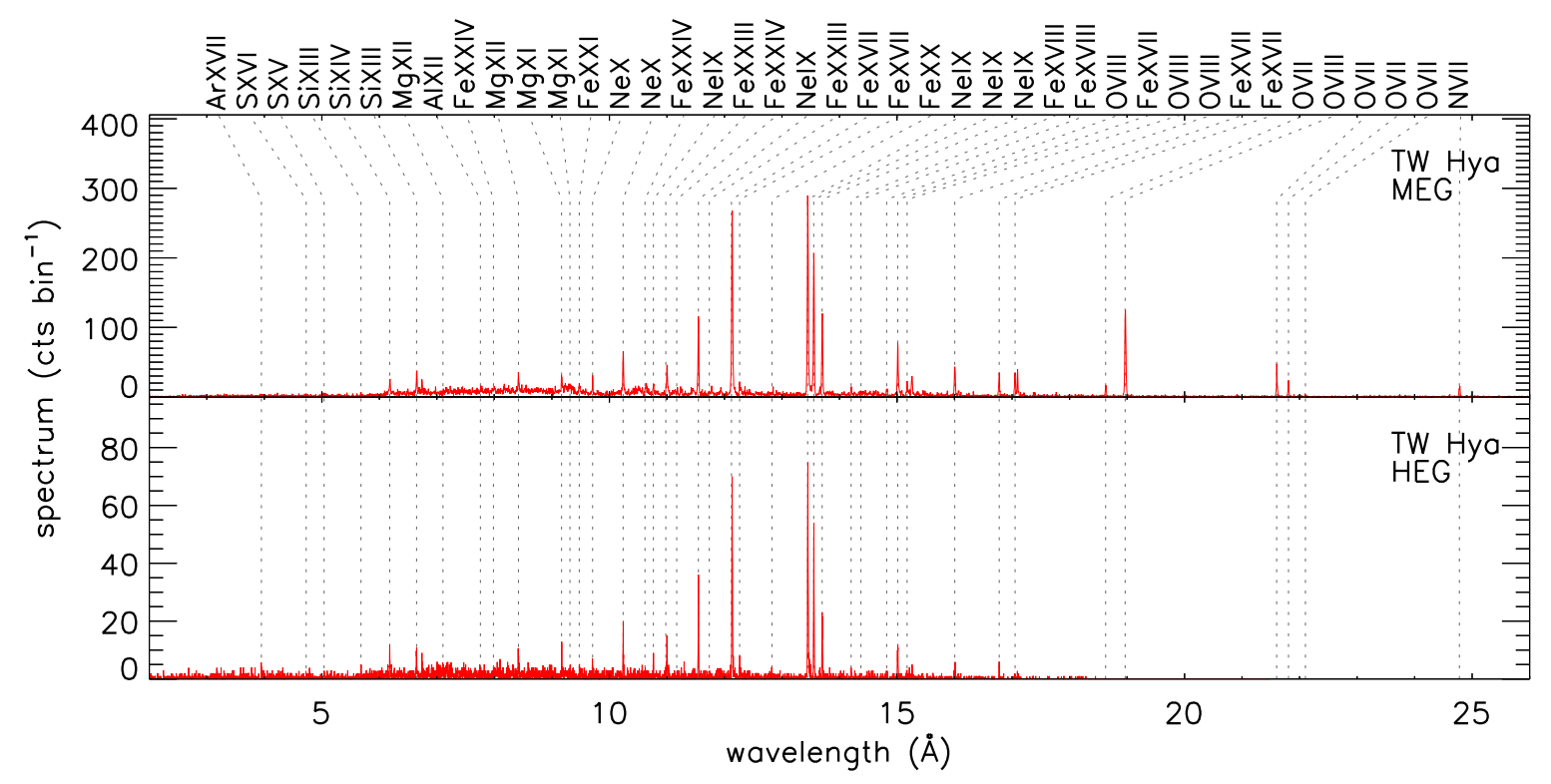

Fig. 1. MEG and HEG spectra of TW Hya obtained during the 2007 observation (ObsID 7435+7436+7437).

$M_{\text {disk }} \gtrsim 0.06 M_{\odot}$ (Bergin et al. 2013), that is viewed almost pole-on $\left(i \approx 7^{\circ}\right.$; Qi et al. 2004). Several gaps and features in the disk indicate that planet formation and/or photoevaporation processes are ongoing (e.g., Hughes et al. 2007; Andrews et al. 2016; Ercolano et al. 2017, and references therein). Because of this orientation, the determination of the rotational period of TW Hya is very controversial. Radial velocity studies provided a periodicity of $3.6 \mathrm{~d}$ (Setiawan et al. 2008), which could be interpreted as the stellar rotational in the hypothesis of variations due to photospheric spots (Huélamo et al. 2008). Conversely, several photometric monitoring campaigns provided different and sparse periodicities $\left(P_{\text {rot }}\right.$ ranging between $\sim 1$ and $\sim 5 \mathrm{~d}$; e.g., Herbst \& Koret 1988; Lawson \& Crause 2005; Siwak et al. 2011, 2014).

TW Hya is still actively accreting matter from its disk with a rate that varies between $\sim 0.5$ and $\sim 2.0 \times 10^{-9} M_{\odot} \mathrm{yr}^{-1}$ (Muzerolle et al. 2000; Curran et al. 2011; Donati et al. 2011). Accretion is certainly regulated by the stellar magnetic field, since TW Hya produces a strong field that is characterized by an average surface intensity of $\sim 1.5 \mathrm{kG}$, but is capable of reaching values of $\sim 3 \mathrm{kG}$ in some magnetic spots (Johns-Krull \& Valenti 2001; Donati et al. 2011). In particular Donati et al. (2011) found that the magnetic configuration is complex and characterized by a strong octupolar component. This, on the one hand, suggests that the magnetosphere is able to disrupt the inner disk at most at distances of $3-4 R_{\star}$ (Donati et al. 2011), and hints that the geometry of the accretion streams is likely more complicated than that of a pure dipolar configuration.

Observational constraints on the accretion geometry provide a complex scenario. Batalha et al. (2002) detected periodic veiling variations and suggested the presence of a hot spot located at low latitudes $\left(\lesssim 20^{\circ}\right)$. Conversely the $\mathrm{H} \beta$ line profile, which is characterized by a slightly redshifted absorption feature, points to a hot spot always visible during stellar rotation, but seen through the accretion stream (Alencar \& Batalha 2002); this scenario is more compatible with high-latitude accretion streams. The different accretion geometries obtained at different epochs, and with different techniques, could be partly reconciled by the spectropolarimetric study presented by Donati et al. (2011). Assuming that the observed variations are due to rotational modulation, these authors suggested that on TW Hya significant accretion can occur both at high and low latitudes, in agreement with the inferred magnetic field configuration. It is worth noting that all the results based on rotational modulation, because of the stellar pole-on view, unknown rotational period, and intrinsic accretion variability, should be considered with caution.

TW Hya, because it is the nearest CTTS, was targeted several times with Chandra and XMM (Kastner et al. 2002; Stelzer \& Schmitt 2004; Ness \& Schmitt 2005; Argiroffi et al. 2009; Brickhouse et al. 2010, 2012). It is X-ray bright, with $L_{\mathrm{X}}=1.4 \times 10^{30} \mathrm{erg} \mathrm{s}^{-1}$. The emission measure distribution ${ }^{2}$ (EMD) of its X-ray emitting plasma clearly shows the presence of two main plasma components: a cool $(T \sim 3 \mathrm{MK})$ and highdensity $\left(n_{\mathrm{e}} \sim(0.6-6.0) \times 10^{12} \mathrm{~cm}^{-3}\right)$ plasma, likely due to accretion, and hotter components $(T \gtrsim 10 \mathrm{MK})$, reasonably related to coronal activity (Kastner et al. 2002; Brickhouse et al. 2010). The clear temperature separation of these two components allows us to infer that $\sim 75 \%$ of the total X-ray luminosity is due to the accretion-related plasma. This fraction increases up to $95 \%$ when only cool X-ray emission lines are considered. The chemical composition of the X-ray emitting plasma in TW Hya is peculiar: it is very rich in neon with respect to other elements. Because the X-ray emitting plasma in TW Hya is related to accretion and hence possibly originates in the inner disk, this plasma could have experienced grain depletion, thereby preferentially maintaining volatile elements, such as neon, that remain in the gas phase (Drake et al. 2005).

\section{Chandra/HETGS data}

To search for Doppler shifts in the X-ray emission from TW Hya, we analyzed the Chandra/HETGS spectrum collected during the Large Observing Project performed in 2007 (ObsID 7435, 7436, and 7437, PI N. Brickhouse), and shown in Fig. 1. Moreover, thanks to the long exposure $(\sim 500 \mathrm{ks})$, this observation yielded a very high signal-to-noise ratio $(\mathrm{S} / \mathrm{N}) \mathrm{X}$-ray spectrum rich in emission lines (Fig. 1). To check for any vari-

2 The EMD is the distribution in temperature of emission measure, EM, defined as the product of the electron density, hydrogen density, and volume. 
Table 1. Nominal properties of the Chandra/HETGS, considering only the positive and negative first-order diffraction spectra.

\begin{tabular}{ccccccccc}
\hline \hline \multirow{2}{*}{ Grating } & & & \multicolumn{2}{c}{ LRF } & & \multicolumn{2}{c}{ Wavelength accuracy } & \\
& $\begin{array}{c}\text { Range } \\
(\AA)\end{array}$ & $\begin{array}{c}\text { Bin size } \\
(\mathrm{m} \AA)\end{array}$ & $\begin{array}{c}F W H M \\
(\mathrm{~m} \AA)\end{array}$ & $\begin{array}{c}\sigma \\
(\mathrm{m} \AA)\end{array}$ & & $\begin{array}{c}\text { Absolute } \\
(\mathrm{m} \AA)\end{array}$ & $\begin{array}{c}\text { Relative } \\
(\mathrm{m} \AA)\end{array}$ & $\begin{array}{c}\text { Eff. Area }(@ 10 \AA) \\
\left(\mathrm{cm}^{2}\right)\end{array}$ \\
\hline HEG & $1.2-15$ & 2.5 & 12 & 5.1 & & 6 & 1.0 & 20 \\
MEG & $1.2-31$ & 5.0 & 23 & 9.8 & & 11 & 2.0 & 51 \\
\hline
\end{tabular}

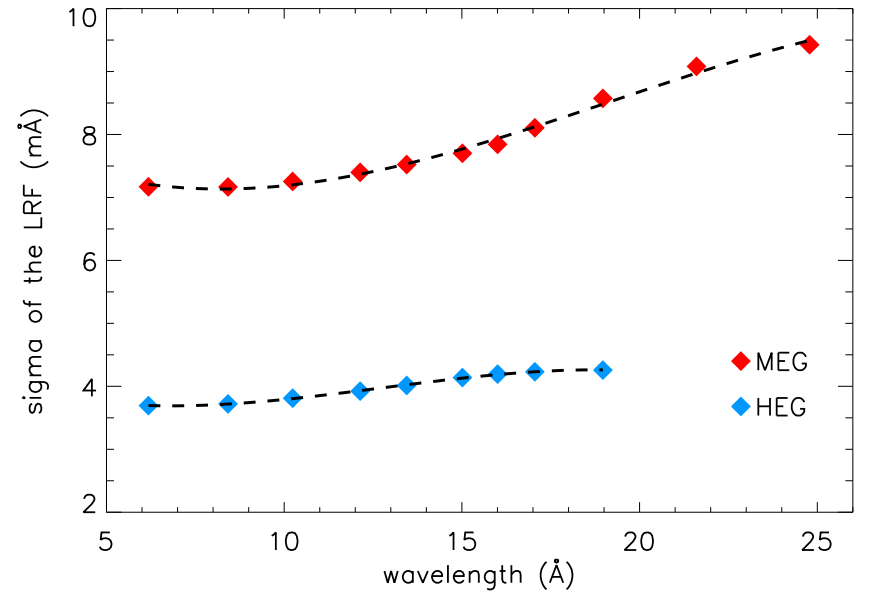

Fig. 2. Predicted Gaussian $\sigma$ of the LRF of the HEG and MEG gratings, computed at the wavelength corresponding to the most intense emission lines. The black dotted curves indicate the best-fit polynomial function adopted to infer the predicted $\sigma$ at each wavelength.

ability in the measured Doppler shift, we also analyzed the shorter Chandra/HETGS observation of TW Hya (exposure time $\sim 50 \mathrm{ks}$, ObsID 5, PI C. Canizares) performed on 2000 July 18, even though its $\mathrm{S} / \mathrm{N}$ is significantly lower.

As reported in Sect. 3.1, looking for Doppler shifts related to plasma motion of $\sim 100 \mathrm{~km} \mathrm{~s}^{-1}$ or lower (depending on the inclination) means pushing the spectral resolution of the Chandra gratings to the limit. Therefore, to test our methods and to check the wavelength accuracy of the instrument, we also considered other Chandra/HETGS observations of active stars as a reference (see Sect. 3.2).

All the MEG and HEG data analyzed in this work have been retrieved from the Grating-Data Archive and Catalog (Huenemoerder et al. 2011).

\subsection{Spectral resolution of the Chandra/HETGS}

The Chandra/HETGS currently provides the highest spectral resolving power available for observations of cosmic sources in the X-ray band. This instrument consists of two gratings, the HEG and the MEG, that, when used with the ACIS-S detector, allow collection of two spectra whose nominal properties ${ }^{3}$ are reported in Table 1. To optimize our methods for Doppler shift measurements, and hence to obtain plasma velocities with the highest possible accuracy, we inspected these nominal properties and how they vary with wavelength.

As a first step we inspected the width of the line response function (LRF), as tabulated in the redistribution matrix function of the two gratings, and how it varies within the wavelength range. To this aim we synthesized the spectra corresponding to individual and isolated emission lines, with no intrinsic

\footnotetext{
3 http://cxc.harvard. edu/proposer/P0G/html/chap8.html
}

broadening, at wavelengths corresponding to the strongest lines in the explored range (i.e., $5<\lambda<25 \AA$ ). We then fitted these simulated line profiles with a Gaussian function. The corresponding $\sigma$ values obtained from these line fits are plotted in Fig. 2. For both HEG and MEG these $\sigma$ are slightly smaller than the corresponding nominal values (see Table 1), and they smoothly increase for increasing wavelengths.

The precision provided by centroid measurements in individual lines is expected to scale as $\sigma / \sqrt{N}$, with $N$ indicating the number of counts in the line. Therefore, in lines with a few hundreds of counts in the MEG grating, a precision of $\sim 10-20 \mathrm{~km} \mathrm{~s}^{-1}$ can be reached in individual line position. By combining results from more lines a higher precision can, in principle, be obtained.

To measure Doppler shifts, it is also fundamental to understand the nominal accuracy of line positions determined by the Chandra/HETGS gratings, which depends on its wavelength calibration. As reported in Table 1, the absolute wavelength accuracy is comparable with the line $\sigma$, hence it corresponds to a velocity of $\sim 100 \mathrm{~km} \mathrm{~s}^{-1}$. The accuracy in relative line position within and between observations is instead smaller by a factor of 5. These nominal accuracies of 1.0 and $2.0 \mathrm{~m} \AA$ correspond to velocities of 20 and $40 \mathrm{~km} \mathrm{~s}^{-1}$, respectively, at wavelengths of $\sim 15 \AA$. Therefore, comparing individual line positions between observations, the Chandra/HETGS allows the tracking of plasma motions down to velocities of a few tens of $\mathrm{km} \mathrm{s}^{-1}$. Several studies (Brickhouse et al. 2001; Chung et al. 2004; Ishibashi et al. 2006) confirmed this accuracy and proved that the Chandra/HETGS is very stable in relative wavelength measurements.

These considerations confirm that the velocities expected for the post-shock plasma in CTTS can be detected with the Chandra gratings, based on relative line positions between different observations.

\subsection{Reference $X$-ray spectra}

As reported in the previous section, the highest wavelength accuracy with Chandra/HETGS data can be obtained comparing line positions between observations. To verify any measured Doppler shift in the X-ray spectrum of TW Hya, we therefore also checked a selected sample of high $\mathrm{S} / \mathrm{N}$ spectra collected with the Chandra/HETGS of active stars without accretion or other expected sources of significant Doppler shifts. In active stars, X-rays are emitted by coronal plasmas, which are located in the outer stellar atmosphere. Therefore it is expected that any Doppler shift in the X-ray spectrum of an active star should yield the same radial velocity as the stellar photosphere. To avoid complications due to variable radial velocity or line broadening, we selected only stars that have negligible radial velocity due to orbital motion and negligible line broadening due to rotation. The selected sample of Chandra/HETGS observations is reported in Table 2, in which we also list the main characteristics of these 
Table 2. Selected sample of stars and their corresponding Chandra/HETGS observation.

\begin{tabular}{|c|c|c|c|c|c|c|c|c|}
\hline \multirow[b]{2}{*}{ Star } & \multicolumn{4}{|c|}{ Chandra Obs. info } & \multicolumn{2}{|c|}{ Stellar properties } & \multirow{2}{*}{$\frac{\text { Earth velocity }}{v_{\mathrm{E}}^{b}\left(\mathrm{~km} \mathrm{~s}^{-1}\right)}$} & \multirow{2}{*}{ ref. ${ }^{c}$} \\
\hline & ObsID & Obs. start & Obs. stop & Exp. time (ks) & $\mathrm{SpTp}$ & $v_{\text {phot }}{ }^{a}\left(\mathrm{~km} \mathrm{~s}^{-1}\right)$ & & \\
\hline TW Hya (1) & $7435+7436+7437$ & $2007-02-15$ & $2007-03-02$ & 468.7 & M0 & 12.4 & 11.8 & 1 \\
\hline TW Hya (2) & 5 & $2000-07-18$ & $2000-07-18$ & 47.7 & M0 & 12.4 & -21.3 & 1 \\
\hline AU Mic & 17 & $2000-11-12$ & $2000-11-12$ & 58.8 & M1 & -4.1 & -28.2 & 2 \\
\hline EV Lac (1) & 1885 & 2001-09-19 & $2001-09-20$ & 100.0 & M4.5 & 0.5 & 2.7 & 2 \\
\hline EV Lac (2) & 10679 & $2009-03-13$ & 2009-03-14 & 95.6 & M4.5 & 0.5 & -4.8 & 2 \\
\hline AD Leo & 2570 & 2002-06-01 & 2002-06-01 & 45.2 & M4.5 & 12.4 & -28.5 & 3 \\
\hline$\beta$ Cet & 974 & $2001-06-29$ & 2001-07-01 & 86.6 & K0 & 13.3 & 27.2 & 4 \\
\hline$\lambda$ And & 609 & $1999-12-17$ & $1999-12-18$ & 81.9 & G8 & 7.0 & -20.2 & 4 \\
\hline$\xi \mathrm{Uma}$ & 1894 & $2000-12-28$ & $2000-12-29$ & 70.9 & $\mathrm{~F} 8.5+\mathrm{G} 2$ & -18.2 & 23.7 & 5 \\
\hline $24 \mathrm{Uma}$ & $2564+3471$ & $2002-03-26$ & $2002-03-30$ & 88.9 & G9 & -27.0 & -17.6 & 6 \\
\hline
\end{tabular}

Notes. ${ }^{(a)}$ Radial photospheric velocity in the heliocentric reference frame. ${ }^{(b)}$ Radial velocity of the Earth along the line of sight, in the heliocentric reference frame, computed at the time corresponding to the middle between the start and the stop of each observation. ${ }^{(c)}$ References for radial photospheric velocities: 1 Setiawan et al. (2008); 2 Bailey et al. (2012); 3 Nidever et al. (2002); 4 Massarotti et al. (2008); 5 Nordström et al. (2004); 6 Gontcharov (2006).

active stars together with their known radial velocity ${ }^{4}$. In this stellar sample, there are also a few multiple systems. In all cases, however, because of the mass ratio and/or because of companion separation, it is known that orbital velocities are negligible $\left(v \lesssim 10 \mathrm{~km} \mathrm{~s}^{-1}\right)$.

For stars with multiple Chandra observations, we considered the HEG and MEG spectra obtained by adding the total exposure time, but only if the different observations were performed approximately at the same epoch (observations 7435, 7436, and 7437 of TW Hya, and observations 2564 and 3471 of 24 UMa, as also reported in Table 2). This is because, only in those cases, the observations were performed in time intervals small enough to have Earth velocity variations at most of a few $\mathrm{km} \mathrm{s}^{-1}$, in the heliocentric reference frame. For the same reason we investigated separately the two observing campaigns of TW Hya, performed on February 2007 and July 2000 and indicated throughout the paper as TW Hya (1) and TW Hya (2), and the two observations of EV Lac, performed on September 2001 and March 2009 and indicated as EV Lac (1) and EV Lac (2). In Table 2 we also report the values of the line-of-sight Earth velocity in the heliocentric reference frame at the epoch of each observation ${ }^{5}$.

\section{Doppler shift measurements and plasma velocity}

We describe in this section the two methods adopted to measure Doppler shifts from the Chandra/HETGS spectra. We followed two different approaches. The first method is based on measuring the positions of a selected sample of lines, and then averaging these velocities to obtain the global velocity corresponding to each observation. Considering the line formation temperatures, we can adjust this procedure so as to consider only lines produced by plasma components at a few MK. The second method is based on fitting the spectrum with parameterized radiative loss models using the CIAO sherpa fitting engine and allowing the line-of-sight velocities for cooler accretion-dominated and hotter emission components to vary independently.

\footnotetext{
4 Throughout the paper we assume that positive radial velocities indicate outward motion (redshift), while negative velocities indicate inward motion (blueshift).

5 Also, we assume positive and negative values for outward and inward motion for the line-of-sight Earth velocity in the heliocentric reference frame, respectively.
}

\subsection{Method 1: individual line positions}

Searching for Doppler shift only in individual selected lines allows us to avoid any line blending problem and to monitor only the velocities of plasma components in small temperature ranges.

\subsubsection{Line selection}

Among the tens of emission lines typically observed in the TW Hya X-ray spectrum (Fig. 1), we first selected a sample of emission lines, including only strong and isolated lines. The selected lines are listed in Table 3, where we also report their rest wavelengths, as predicted by the APED atomic databases (Smith et al. 2001), and their maximum formation temperature.

Although we are only interested in single lines, we also included some resonance doublets of $\mathrm{H}$-like ions in our sample (indicated in Table 3 with the same index number, but different letters). Considering the spectral resolution of the HEG and MEG gratings, the two components of each doublet are not resolved, but their wavelength difference is not negligible. This is not an issue for the line position determination since the relative position and relative intensity (exactly equal to $2: 1$ in the optically thin emission regime) of the two contributions are known. Therefore, even if these doublets are not strictly isolated single lines, the total doublet profile is perfectly known.

Accretion shocks in CTTS can generate plasma whose temperatures are at most a few MK. A Doppler shift with respect to the stellar photosphere is therefore expected only for the $\mathrm{X}$-ray lines produced at these temperatures. The maximum formation temperature $T_{\max }$ of the selected lines ranges from 2 up to $16 \mathrm{MK}$. We considered the EMD of TW Hya (model $C$ from Brickhouse et al. 2010) to better constrain the origin of each selected line; this EMD is characterized by a sharp peak at $T \approx 2.5 \mathrm{MK}$ ascribed to the accretion shock, and by a large peak centered at $T \approx 13 \mathrm{MK}$ ascribed to coronal plasma (Fig. 3). This EMD, together with the emissivity functions $\epsilon(T)$ of each selected line (also shown in Fig. 3, normalized to their maximum), allowed us to compute the expected contributions of accretion and coronal components to the flux of each line. These relative contributions are reported in Table 3. We therefore selected the subset of cool lines (labeled with "c" in Table 3 and with red curves in Fig. 3). All these cool lines have $T_{\max }<4.5 \mathrm{MK}$ and 
Table 3. Emission lines selected for Doppler shift measurements.

\begin{tabular}{|c|c|c|c|c|c|c|}
\hline \multirow[t]{2}{*}{ Index $^{a}$} & \multirow{2}{*}{$\begin{array}{l}\lambda^{b} \\
(\AA)\end{array}$} & \multirow[t]{2}{*}{ Elem. } & \multirow{2}{*}{$\begin{array}{c}T_{\max } \\
(\mathrm{MK})\end{array}$} & \multicolumn{2}{|c|}{ Flux contrib. ${ }^{c}$} & \multirow{2}{*}{$\begin{array}{l}\text { Line } \\
\text { subset }\end{array}$} \\
\hline & & & & Accr. & Cor. & \\
\hline $1 \mathrm{a}$ & 6.1804 & Si XIV & 15.8 & 0.00 & 1.00 & $\mathrm{~h}$ \\
\hline $1 b$ & 6.1858 & Si XIV & 15.8 & 0.00 & 1.00 & $\mathrm{~h}$ \\
\hline 2 & 6.6479 & Si XIII & 10.0 & 0.01 & 0.99 & $\mathrm{~h}$ \\
\hline 3 & 6.7403 & Si XIII & 10.0 & 0.02 & 0.98 & $\mathrm{~h}$ \\
\hline $4 a$ & 8.4192 & Mg XII & 10.0 & 0.00 & 1.00 & $\mathrm{~h}$ \\
\hline $4 b$ & 8.4246 & Mg XII & 10.0 & 0.00 & 1.00 & $\mathrm{~h}$ \\
\hline 5 & 9.1687 & Mg XI & 6.3 & 0.31 & 0.69 & $\mathrm{~h}$ \\
\hline $6 a$ & 9.7080 & $\mathrm{NeX}$ & 6.3 & 0.07 & 0.93 & $\mathrm{~h}$ \\
\hline $6 b$ & 9.7085 & $\mathrm{NeX}$ & 6.3 & 0.07 & 0.93 & $\mathrm{~h}$ \\
\hline $7 a$ & 10.2385 & $\mathrm{NeX}$ & 6.3 & 0.09 & 0.91 & $\mathrm{~h}$ \\
\hline $7 b$ & 10.2396 & $\mathrm{NeX}$ & 6.3 & 0.09 & 0.91 & $\mathrm{~h}$ \\
\hline 8 & 13.6990 & Ne IX & 4.0 & 0.96 & 0.04 & c \\
\hline 9 & 15.0140 & Fe XVII & 5.0 & 0.73 & 0.27 & $\mathrm{~h}$ \\
\hline $10 \mathrm{a}$ & 16.0055 & O VIII & 3.2 & 0.92 & 0.08 & $\mathrm{c}$ \\
\hline $10 \mathrm{~b}$ & 16.0067 & O VIII & 3.2 & 0.92 & 0.08 & $\mathrm{c}$ \\
\hline 11 & 16.7800 & Fe XVII & 5.0 & 0.77 & 0.23 & $\mathrm{~h}$ \\
\hline 12 & 17.0510 & Fe XVII & 5.0 & 0.76 & 0.24 & $\mathrm{~h}$ \\
\hline 13 & 17.0960 & Fe XVII & 5.0 & 0.78 & 0.22 & $\mathrm{~h}$ \\
\hline $14 \mathrm{a}$ & 18.9671 & O VIII & 3.2 & 0.94 & 0.06 & $\mathrm{c}$ \\
\hline $14 b$ & 18.9725 & O VIII & 3.2 & 0.94 & 0.06 & $\mathrm{c}$ \\
\hline 15 & 21.6015 & O VII & 2.0 & 1.00 & 0.00 & $\mathrm{c}$ \\
\hline
\end{tabular}

Notes. ${ }^{(a)}$ Lines indicated with the same index number, but different letters, are the two components of H-like resonance doublets. ${ }^{(b)}$ APED predicted wavelengths. ${ }^{(c)}$ Fractional contributions to individual line fluxes due to accretion and corona components, on the basis of the model $C$ obtained by Brickhouse et al. (2010). ${ }^{(d)}$ "c" and " $h$ " indicate whether the line has been included in the cool or hot line subsample.

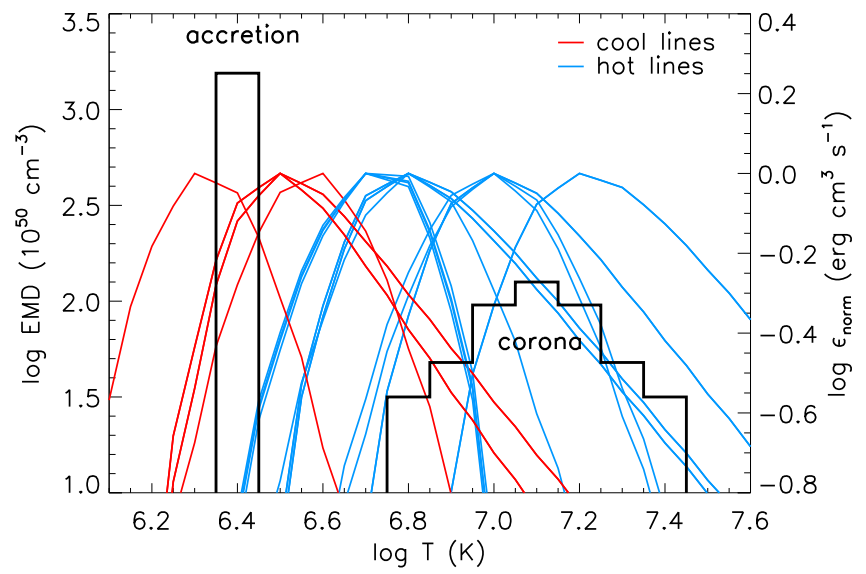

Fig. 3. EMD (in black) of the X-ray emitting plasma in TW Hya, composed by an accretion component and a coronal component (model C derived by Brickhouse et al. 2010). Red and blue curves indicate the normalized emissivity function $\epsilon_{\text {norm }}$ of the cool and hot selected lines.

any coronal contribution to their flux is expected to be less than $10 \%$. Therefore these lines should allow us to probe the velocity of the plasma heated in the accretion shock. The remaining lines, indicated as hot lines (labeled with " $h$ " in Table 3 and with blue curves in Fig. 3) have maximum formation temperatures higher than $4.5 \mathrm{MK}$, and a non-negligible coronal contribution to their flux. Some of these hot lines, especially the Fe XVII lines, have significant contributions from both accretion and coronal plasma components. Their Doppler shifts might therefore be an average between these two plasma components.
Considering the full line set we checked the effect of possible blends due to other weak emission lines, which were not detectable individually but could skew the line position estimation. To this aim, we checked that the expected contribution of weak and unresolved lines in the wavelength regions of the lines selected for analysis was lower than $\sim 5 \%$. Therefore, blending problems due to unresolved lines are expected to be negligible in the determination of the centroid position of each line.

\subsubsection{Line position measurement}

We determined the position of the selected emission lines by fitting separately each line profile in the HEG and MEG spectra, obtained by adding positive and negative first order diffraction spectra. We performed the fit in a small wavelength interval of $\sim 0.1-0.2 \AA$, centered on the predicted line wavelength, for each grating and for each line. For some observations, some lines in the HEG grating are almost undetected. We therefore performed the line fit only when a minimum number of 5 counts were gath$\operatorname{ered}^{6}$ in the selected wavelength interval. As a best-fit function we assumed a Gaussian profile plus a constant, the latter serving to represent the continuum level. In the cases of the H-like resonance doublets, we fitted the observed profiles with two Gaussians, whose displacement and relative intensity are fixed to the known theoretical values. In all cases, to minimize the errors on the measurements of the line positions, we performed the fits fixing the $\sigma$ of the Gaussian profile to the value predicted by the LRF and reported in Sect. 3.1. The observed and fitted line profiles are shown in Figs. A.1 and A.2.

\subsubsection{Determination of the radial velocity of the X-ray emitting plasma}

For each fitted line, we determined the velocity corresponding to the wavelength shift with respect to the predicted wavelength rest value. The velocities obtained are reported in Table A.1 and plotted in Fig. 4. These velocities are computed with respect to the Chandra satellite reference frame.

Considering all the Chandra/HETGS spectra adopted as a reference, the velocities provided from different lines do not show any systematic pattern across the explored wavelength range. The only exception to this lack of pattern is the Fe XVII line at $16.78 \AA$ (denoted with an open symbol in Fig. 4); in all the observations this line is always significantly and systematically blueshifted, if compared to the other lines of the same observation. We therefore excluded this line from our subsequent analysis, assuming that there is probably some issue in its predicted rest wavelength.

Since the other lines do not show any systematic trend, we assumed that the individual line velocities are random fluctuations of the X-ray plasma velocity. Therefore, for each observation we inferred the plasma velocity by computing the weighted average of the velocities obtained from individual lines, considering separately the cool and hot line subsamples, as reported in Sect. 4.1.1 and as indicated in Table 3. These cool and hot

\footnotetext{
6 The value of 5 cts threshold, set for very weak lines, does not significantly affect our procedure. The positions of marginally detected lines are in any case characterized by large uncertainty, hence these lines do not significantly affect the final determination of the plasma radial velocity obtained by a weighted average (see Sect. 4.1.3).
} 


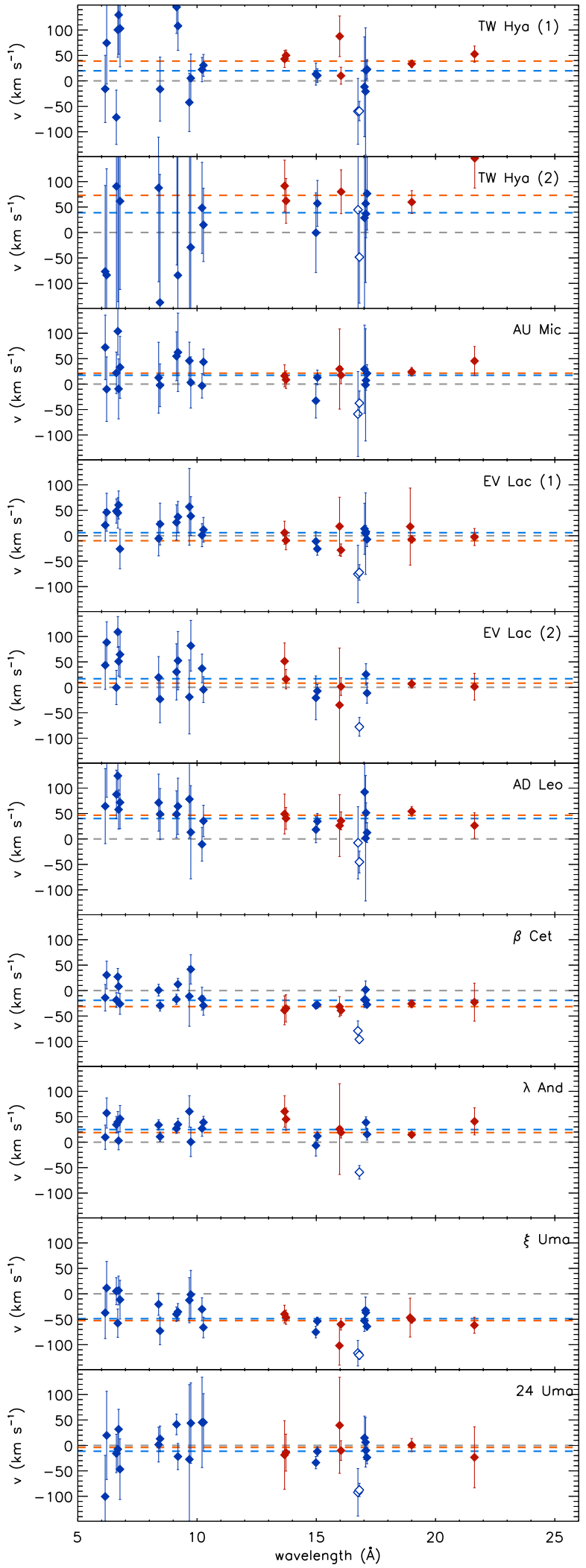

Fig. 4. Velocity obtained from each fitted line, for each inspected spectrum, with respect to the Chandra satellite reference frame. The red and blue diamonds indicate lines included in the cool and hot line sample, respectively, and errors refer to the $1 \sigma$ level. The red and blue dashed lines correspond to the average velocities, $v_{\mathrm{Xc}}$ and $v_{\mathrm{Xh}}$, obtained for the cool and hot line sample. The velocity measured for the Fe XVII line at $16.78 \AA$, not included in the average velocity computation, is indicated with an open diamond. To clarify, we included a small wavelength offset in those points corresponding to the same line fitted in the two gratings. average velocities ${ }^{7}, v_{\mathrm{Xc}}$ and $v_{\mathrm{Xh}}$, are reported in Table A.1, and plotted as horizontal dashed lines in Fig. 4.

We performed further checks to investigate whether the final average velocities, $v_{\mathrm{Xc}}$ and $v_{\mathrm{Xh}}$, depend on including or not the HEG spectra (that are of higher spectral resolution but characterized by significantly lower $\mathrm{S} / \mathrm{N}$ and provide significantly larger uncertainties in the line velocities), and/or depend on considering separately the +1 and -1 diffraction orders. We found that the average velocities, $v_{\mathrm{Xc}}$ and $v_{\mathrm{Xh}}$, do not change significantly when computed with various approaches.

\subsection{Method 2: spectral model parameter estimation}

The second method we used for Doppler shift measurements consists in modeling the whole X-ray spectrum at once. This approach offers the advantage, at least in principle, of implicitly including all of the velocity information contained in the data into the analysis.

We examined the long TW Hya observation, obtained in 2007, indicated here as TW Hya (1), and composed by three individual exposures of $\approx 150 \mathrm{ks}$ each (ObsIDs 7435, 7436 and 7437). We modeled them both separately and combined.

We considered HEG and MEG spectra in the wavelength ranges of $1.5-18 \AA$ and $2-26 \AA$, respectively. We used the sherpa fitting engine in CIAO version 4.9 with APEC models, and employed the model $C$ coronal and accretion plasma EMD described by Brickhouse et al. (2010), reported here in Fig. 3. This EMD is characterized by a fixed temperature component, at $2.5 \mathrm{MK}$, representing the post-shock plasma, and a hotter component (a Gaussian-broadened $\delta$ function) centered on $13 \mathrm{MK}$. One difference with respect to the analysis of Brickhouse et al. (2010) is that we allowed the centroid temperature of this latter component to vary during spectral fitting. We also allowed the $\mathrm{Ne}$ abundance in this component to vary independently of other metals. As also noted by Brickhouse et al. (2010), the abundances of metals in the accreting gas and absorption due to interstellar and ambient gas are not independently well constrained by the data. The absorption due to ambient gas is also probably very complex and non-uniform. Since here we are primarily concerned with understanding Doppler shifts rather than in building an accurate picture of the coronal and accretion plasma characteristics, we fixed the absorption component $N_{\mathrm{H}}$ to the single value of $10^{21} \mathrm{~cm}^{-2}$, as adopted by Brickhouse et al. (2010). Additionally, we fixed the $\mathrm{Ne}$ and other metal abundances in the accretion component at 1.2 and 0.2 times the solar values of Anders \& Grevesse (1989), respectively, following Drake et al. (2005) and also guided by the Brickhouse et al. (2010) results. No attempt was made to match the ratios of the density sensitive He-like ions that were first pointed out, by Kastner et al. (2002) and Stelzer \& Schmitt (2004), as showing higher densities in TW Hya than in non-accreting magnetically active stars.

We list in Table 4 the model fit results, together with the velocities of cool and hot plasma components, reporting both the parameters obtained from combined or individual observation analysis. The individual model component normalization parameter ("Norm" in Table 4) depends on plasma emission measure and source distance. In the case of simultaneous modeling of different observations, independent multiplicative constants were introduced to allow the global normalization of the model to vary among the different observation segments.

7 These are always the velocities with respect to the Chandra satellite reference frame. 
Table 4. Spectral model parameter estimation for TW Hya.

\begin{tabular}{|c|c|c|c|c|c|c|}
\hline \multirow[b]{2}{*}{ Comp. } & \multirow[b]{2}{*}{ Parameter } & \multicolumn{5}{|c|}{ Observation } \\
\hline & & TW Hya $(1)^{a}$ & TW Hya (7435) & TW Hya (7436) & TW Hya (7437) & TW Hya $(7435+7436)$ \\
\hline & $N_{\mathrm{H}}^{b}\left(10^{20} \mathrm{~cm}^{-2}\right)$ & 10 & 10 & 10 & 10 & 10 \\
\hline \multirow[t]{5}{*}{ Cool } & $T^{b}(\mathrm{MK})$ & 2.5 & 2.5 & 2.5 & 2.5 & 2.5 \\
\hline & $\mathrm{Ne}^{b}\left(\mathrm{Ne}_{\odot}\right)$ & 1.2 & 1.2 & 1.2 & 1.2 & 1.2 \\
\hline & $\mathrm{Fe}^{b}\left(\mathrm{Fe}_{\odot}\right)$ & 0.2 & 0.2 & 0.2 & 0.2 & 0.2 \\
\hline & $v_{\mathrm{Xc}}\left(\mathrm{km} \mathrm{s}^{-1}\right)$ & $44 \pm 2$ & $40 \pm 4$ & $47 \pm 4$ & $44 \pm 4$ & $44 \pm 3$ \\
\hline & $\operatorname{Norm}^{c}\left(10^{4}\right)$ & $76.7 \pm 1.3$ & $83.6 \pm 2.1$ & $102 \pm 2.3$ & $87.6 \pm 2.2$ & $81.3 \pm 1.5$ \\
\hline \multirow[t]{7}{*}{ Hot } & $T(\mathrm{MK})$ & $11.59 \pm 0.15$ & $11.13 \pm 0.27$ & $11.54 \pm 0.24$ & $12.02 \pm 0.25$ & $11.36 \pm 0.18$ \\
\hline & $\mathrm{Ne}\left(\mathrm{Ne}_{\odot}\right)$ & $1.38 \pm 0.06$ & $1.38 \pm 0.12$ & $1.38 \pm 0.11$ & $1.37 \pm 0.10$ & $1.39 \pm 0.08$ \\
\hline & $\mathrm{Fe}\left(\mathrm{Fe}_{\odot}\right)$ & $0.086 \pm 0.004$ & $0.084 \pm 0.008$ & $0.085 \pm 0.007$ & $0.087 \pm 0.007$ & $0.085 \pm 0.005$ \\
\hline & $v_{\mathrm{Xh}}\left(\mathrm{km} \mathrm{s}^{-1}\right)$ & $25 \pm 6$ & $9.4 \pm 10$ & $25 \pm 9$ & $37 \pm 9$ & $19 \pm 7$ \\
\hline & $\operatorname{Norm}^{c}\left(10^{4}\right)$ & $1.51 \pm .04$ & $1.46 \pm 0.07$ & $1.88 \pm 0.07$ & $2.01 \pm 0.08$ & $1.46 \pm 0.05$ \\
\hline & 7436 Norm $^{d}$ & $1.28 \pm 0.02$ & $\ldots$ & $\ldots$ & $\ldots$ & $1.28 \pm 0.02$ \\
\hline & 7437 Norm $^{d}$ & $1.28 \pm 0.02$ & $\ldots$ & $\ldots$ & $\ldots$ & $\ldots$ \\
\hline
\end{tabular}

Notes. All the errors refer to the $1 \sigma$ level. ${ }^{(a)}$ This column reports the results obtained considering the combination of the ObsID 7435+7436+7437, we labeled it as TW Hya (1) in agreement with the rest of the work. ${ }^{(b)}$ Parameter fixed. ${ }^{(c)}$ Normalization parameter in units of $10^{-14} /\left(4 \pi d^{2}\right) \int n_{\mathrm{e}} n_{\mathrm{H}} \mathrm{d} V$, where $d$ is the distance to the source $(\mathrm{cm}), n_{\mathrm{e}}$ is the plasma electron density $\left(\mathrm{cm}^{-3}\right)$, and $n_{\mathrm{H}}$ is the plasma hydrogen density $\left(\mathrm{cm}^{-3}\right) .{ }^{(d)}$ Global normalization constant applied multiplicatively to the whole model (both hot and cool components) in simultaneous fit.

Table 5. Radial velocities of the cool and hot X-ray emitting plasmas in the Chandra $\left(v_{\mathrm{Xc}}\right.$ and $\left.v_{\mathrm{Xh}}\right)$ and in the Stellar $\left(v_{\mathrm{Xc}}^{\star}\right.$ and $\left.v_{\mathrm{Xh}}^{\star}\right)$ reference frames.

\begin{tabular}{|c|c|c|c|c|c|c|}
\hline Star & $\begin{array}{l}v_{\mathrm{E}}^{a} \\
\text { (Heliocentri }\end{array}$ & $\begin{array}{l}v_{\text {phot }} b \\
\text { Ref. frame) }\end{array}$ & $\begin{array}{l}v_{\mathrm{Xc}} \\
\text { (Chandra }\end{array}$ & $\begin{array}{c}v_{\mathrm{Xh}} \\
\text { ef. frame) }\end{array}$ & $\begin{aligned} & v_{\mathrm{X} c}^{\star} \\
&(\text { Stellar Re }\end{aligned}$ & $\begin{array}{r}v_{\mathrm{Xh}}^{\star} \\
\text { f. frame) }\end{array}$ \\
\hline \multicolumn{7}{|c|}{ Method 1: individual line positions } \\
\hline TW Hya (1) & 11.8 & 12.4 & $38.9 \pm 5.1$ & $19.9 \pm 6.9$ & $38.3 \pm 5.1$ & $19.3 \pm 6.9$ \\
\hline TW Hya (2) & -21.3 & 12.4 & $73.1 \pm 16.4$ & $38.8 \pm 23.2$ & $39.3 \pm 16.4$ & $5.1 \pm 23.2$ \\
\hline AU Mic & -28.2 & -4.1 & $21.5 \pm 6.5$ & $17.4 \pm 7.1$ & $-2.6 \pm 6.5$ & $-6.7 \pm 7.1$ \\
\hline EV Lac (1) & 2.7 & 0.5 & $-9.9 \pm 5.3$ & $5.9 \pm 5.3$ & $-7.8 \pm 5.3$ & $8.1 \pm 5.3$ \\
\hline EV Lac (2) & -4.8 & 0.5 & $8.2 \pm 6.6$ & $16.9 \pm 6.9$ & $2.8 \pm 6.6$ & $11.5 \pm 6.9$ \\
\hline AD Leo & -28.5 & 12.4 & $46.6 \pm 7.2$ & $40.1 \pm 7.6$ & $5.7 \pm 7.2$ & $-0.8 \pm 7.6$ \\
\hline$\beta$ Cet & 27.2 & 13.3 & $-31.5 \pm 5.4$ & $-19.1 \pm 2.2$ & $-17.6 \pm 5.4$ & $-5.2 \pm 2.2$ \\
\hline$\lambda$ And & -20.2 & 7.0 & $18.9 \pm 4.7$ & $24.8 \pm 3.2$ & $-8.3 \pm 4.7$ & $-2.4 \pm 3.2$ \\
\hline$\xi \mathrm{Uma}$ & 23.7 & -18.2 & $-52.7 \pm 4.6$ & $-48.9 \pm 3.4$ & $-10.8 \pm 4.6$ & $-7.0 \pm 3.4$ \\
\hline $24 \mathrm{Uma}$ & -17.6 & -27.0 & $-3.9 \pm 9.9$ & $-11.4 \pm 4.7$ & $5.5 \pm 9.9$ & $-2.0 \pm 4.7$ \\
\hline \multicolumn{7}{|c|}{ Method 2: spectral model parameter estimation } \\
\hline TW Hya (1) & 11.8 & 12.4 & $44.0 \pm 2.0$ & $25.0 \pm 6.0$ & $43.4 \pm 2.0$ & $24.4 \pm 6.0$ \\
\hline
\end{tabular}

Notes. All the velocities are in $\mathrm{km} \mathrm{s}^{-1}$. All the errors refer to the $1 \sigma$ level. ${ }^{(a)}$ Radial velocity of the Earth, computed at the time corresponding to the middle between the start and the stop of each observation. ${ }^{(b)}$ Radial photospheric velocity of each star.

Considering the results obtained from individual observations of TW Hya, we found a slightly different velocity for the hot component of ObsID 7437 than for the other two observations. We therefore also performed simultaneous parameter estimation for just ObsID 7435 and 7436 spectra, excluding ObsID 7437, as shown in Table 4.

\subsection{Velocity reference frame}

Both the methods applied provide the bulk velocities of the cool and hot plasma components, $v_{\mathrm{Xc}}$ and $v_{\mathrm{Xh}}$, averaged over the whole exposure time of the inspected observations and computed with respect to the Chandra satellite reference frame. During its orbit, the satellite reaches velocities of at most $\sim 1-2 \mathrm{~km} \mathrm{~s}^{-1}$ with respect to the Earth. Such velocities are negligible with respect to both the errors on the average velocities computed $\left(\sim 5-10 \mathrm{~km} \mathrm{~s}^{-1}\right)$ and the velocities that we aim to measure (tens of $\mathrm{km} \mathrm{s}^{-1}$ ). We therefore assume that the velocity measured with respect to the satellite reference frame is the same as that with respect to the Earth. Therefore, taking into account the line-ofsight Earth velocity in the heliocentric reference frame at the epoch of each observation $v_{\mathrm{E}}$ (listed in Table 2), the X-ray plasma velocity in the stellar reference frame, $v_{\mathrm{X}}^{\star}$, can be computed as $v_{\mathrm{X}}+v_{\mathrm{E}}-v_{\text {phot }}$. All these velocities are listed in Table 5.

\section{Results}

We show in Fig. 5 the cool and hot plasma velocities for each star, $v_{\mathrm{Xc}}^{\star}$ and $v_{\mathrm{Xh}}^{\star}$, with respect to the star itself. The first conclusion is that the two methods applied to TW Hya (1) to measure $v_{\mathrm{Xc}}^{\star}$ and $v_{\mathrm{X} h}^{\star}$ provide compatible results, indicating that the measured velocities do not depend on the methods. In the subsequent discussion, we refer to the $v_{\mathrm{Xc}}^{\star}$ and $v_{\mathrm{Xh}}^{\star}$ values obtained 
C. Argiroffi et al.: Redshifted X-rays from TW Hya: A low-latitude accretion spot

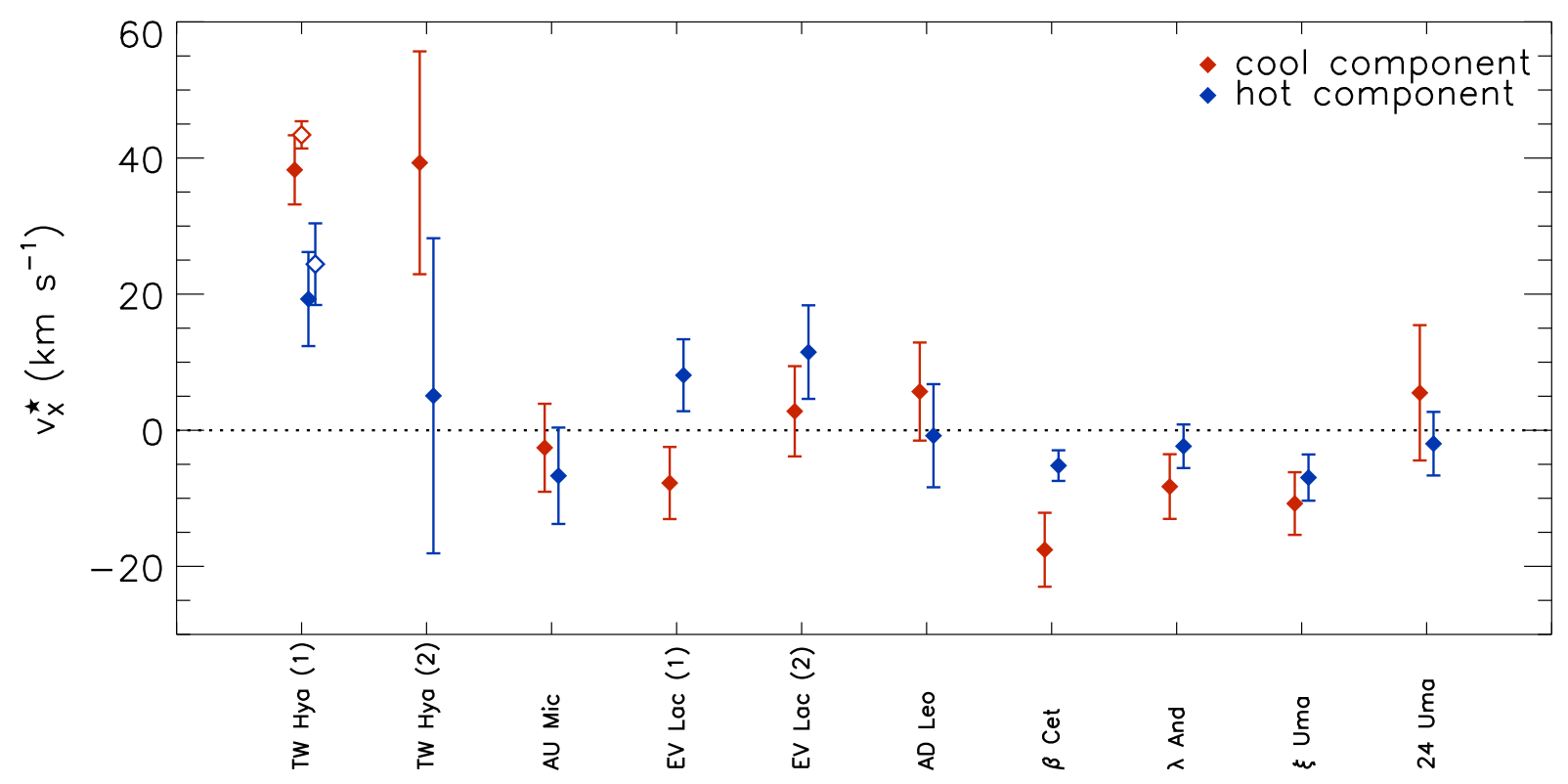

Fig. 5. Cool and hot X-ray emitting plasma velocities $v_{\mathrm{X}}^{\star}$ in the stellar reference frame with errors at the $1 \sigma$ level. Filled symbols indicate velocities obtained with method 1 (individual line positions), open symbols indicate velocities obtained with method 2 (spectral model parameter estimation).

with method 1 , although we stress that velocities obtained with methods 1 and 2 are in excellent agreement.

Inspecting the results obtained for the set of reference stars, where the X-ray emission originates only from coronal plasma, we found that both the cool and hot plasma components provide velocities compatible with zero. The only marginal exception is the cool plasma associated with $\beta$ Cet, which shows a blueshifted velocity with respect to the stellar reference frame of $-17.6 \pm 5.4 \mathrm{~km} \mathrm{~s}^{-1}$, significant at $3.2 \sigma$ level. However, considering the entire set of reference stars, their cool plasma velocities are marginally, but systematically, blueshifted with respect to the photosphere, with a mean value of $\sim-4 \pm 3 \mathrm{~km} \mathrm{~s}^{-1}$. Taking into account this small offset would also reconcile also the $\beta$ Cet case. We can therefore conclude that the X-ray emitting plasma in purely coronal sources displays on average the same velocity as the underlying stellar photosphere. This finding, on the one hand, confirms the reliability of the adopted procedures to measure the X-ray emitting plasma velocity and, on the other hand, verifies the accuracy of the wavelength calibration of the Chandra gratings.

TW Hya clearly shows a different behavior. For the long exposure observation (labeled as TW Hya (1)) we found with both our methods that the cool plasma component is significantly redshifted with respect to the stellar photosphere. Method 1 provides a receding velocity of this cool plasma component of $38.3 \pm 5.1 \mathrm{~km} \mathrm{~s}^{-1}$. A perfectly compatible value, $42.4 \pm$ $2.0 \mathrm{~km} \mathrm{~s}^{-1}$, comes from method 2 .

The TW Hya (1) observation is composed of three observing segments, of $\sim 150 \mathrm{ks}$ each, spread over a time period of a few days (see Table 2). The receding velocity of the cool plasma component does not change significantly considering individual observing segments (see Table 4). Moreover, we also found the same receding velocity for the short exposure observation, TW Hya (2), performed a few years before (despite the lower significance owing to a lower $\mathrm{S} / \mathrm{N}$ ratio of this spectrum).

The velocity of the hot plasma component on TW Hya, determined with both methods 1 or $2,(19.3 \pm 6.9$ and $24.4 \pm 6.0$, respectively) is only marginally consistent with zero velocity offset. This could reflect the adopted velocity determination method, which is based on line position. In fact, as already argued in Sect. 4.1.1, the velocity of the hot plasma component is likely an average between both the accretion and coronal components. To confirm this assessment, we simulated synthetic Chandra/HETGS spectra of TW Hya assuming that its $\mathrm{X}$-ray emission is due to an accretion and a coronal component (model $C$, presented by Brickhouse et al. 2010, and shown in Fig. 3), and supposing that the accretion component is receding with respect to the photosphere, while the coronal plasma is fixed. If we apply the same procedure to the total synthetic spectrum that we applied to the real TW Hya spectrum (method 1, Sect. 4.1), the hot plasma component appears slightly redshifted with respect to the assumed zero velocity of the corona. As predicted, this happens because several emission lines, and almost all the strong Fe XVII lines, have significant contributions from both accretion and coronal components, and hence show a global redshift that is an average between the two assumed input velocities $^{8}$. Nevertheless, it could be that the EMD of TW Hya is not actually composed of two well-separated components (such as those taken as a reference in this work, Fig. 3), but could also have non-negligible amounts of plasma at $T \sim 4-5 \mathrm{MK}$ (i.e., $\log T \sim 6.6-6.7$; as found by Argiroffi et al. 2009) that comes from accretion and hence also produces, in the whole X-ray spectrum, redshifted features at temperatures higher than those predicted by the EMD model assumed here.

In the following discussion, focused on the redshift observed for the cool plasma associated with TW Hya, we interpret this redshift as due to a dominant cool plasma component moving as a whole with the observed velocity. We inspected the profiles of the strongest emission lines in the TW Hya (1) HEG spectrum, considering separately +1 and -1 diffraction orders, to validate this hypothesis of a single velocity component. We fitted these lines with the $\sigma$ left as a free parameter, and we verified that the best-fit values for $\sigma$ were in all cases compatible with the values predicted by the LRF. Therefore in the TW Hya (1) line profile there is no evidence of line broadening, and hence of the presence of a distribution of velocities. Considering the intrinsic

8 The LRF of HEG and MEG gratings does not allow us to resolve the two velocity components in individual line profiles. 
spectral resolution of the Chandra gratings, however, we cannot rule out that the observed redshift could come from a mixture of plasma components receding with velocities differing by a few tens of $\mathrm{km} \mathrm{s}^{-1}$.

\section{Discussion}

Inspecting the X-ray emission from TW Hya, we detected a redshift, with respect to the photosphere, in the plasma components at $\sim 2-4 \mathrm{MK}$. This result provides important constraints on the origin of this plasma component and on the geometry of the accretion streams on TW Hya.

\subsection{Cool plasma origin in CTTS}

The nature of the cool, high-density plasma component observed in CTTS has been the subject of considerable debate. On the one hand, the high density $\left(n_{\mathrm{e}} \sim 10^{12} \mathrm{~cm}^{-3}\right)$ and cool temperature $(T \sim 1-4 \mathrm{MK})$ of this plasma have been the main indications that this material is heated in the accretion shock (Kastner et al. 2002; Stelzer \& Schmitt 2004; Schmitt et al. 2005; Günther et al. 2006; Argiroffi et al. 2007, 2011; Huenemoerder et al. 2007). This scenario was then supported by further constraints: the cool plasma EMD is sharply peaked around $T \sim 3 \mathrm{MK}$ (Brickhouse et al. 2010) as predicted by HD models (Sacco et al. 2008, 2010); some strong emission lines emitted by this cool plasma component show a nonnegligible optical depth (Argiroffi et al. 2009) and display rotational modulation (Argiroffi et al. 2012). On the other hand, other aspects do not fit easily into this scenario. Güdel et al. (2007) did not detect any high-density plasma component from the CTTS T Tauri. Güdel \& Telleschi (2007) noted that the soft X-ray excess shown by CTTS, with respect to non-accreting young stars, does not correlate with UV accretion indicators. Moreover, Brickhouse et al. (2010) found that both the density versus temperature pattern (characterized by increasing density for increasing temperature) and the volumes of the cool plasma components of TW Hya are not reconcilable with the hypothesis of a plasma heated in the accretion shock. Based on the above results, it was suggested that, in addition to plasma heated in the accretion shock, the cool plasma in CTTS could also be composed of coronal structures that are modified, cooled, or fed by accreted material (Güdel \& Telleschi 2007; Brickhouse et al. 2010; Schneider et al. 2017).

One-dimensional HD modeling of the accretion shock region indicates that on average the hot post-shock plasma is indeed characterized by high-density and relatively cool temperature compared with the X-ray emitting corona, similar to that observed (Sacco et al. 2008, 2010). Detailed two-dimensional MHD models show that the hot post-shock region can be composed of independent fibrils, and, because of time-dependent thermal instabilities, can have a composite density versus temperature structure (Bonito et al. 2014). Moreover, when the effect of local absorption due to the pre-shock material is taken into account, the apparent density versus temperature structure inferred from the emerging X-ray spectrum shows a pattern analogous to that observed (Bonito et al. 2014). Two-dimensional MHD models (Orlando et al. 2010) show that any flow of accreted material from the flux tube into the surrounding corona is prevented, unless the local magnetic field is weak, i.e., even a moderate magnetic field of $\sim 200 \mathrm{G}$ provides a plasma $\beta \sim 1$ for a plasma density of $\sim 10^{13} \mathrm{~cm}^{-3}$. Typical values of surface magnetic fields of CTTS are significantly stronger $(B \sim 1-3 \mathrm{kG}$; e.g., Johns-Krull 2007, and references therein), in agreement with magnetospheric accretion that requires accretion tubes to have strong magnetic fields to be able to load and guide inner disk matter.

One of the most important predictions for the plasma heated in the accretion shock, confirmed by detailed HD and MHD simulations, is that it should have a downward bulk velocity within the stellar atmosphere (Sacco et al. 2008, 2010; Orlando et al. 2010, 2013; Bonito et al. 2014). Therefore, depending on the inclination under which the shock region is observed, X-ray lines emitted by post-shock plasma are expected to be redshifted. We show in this work that the X-ray emission lines from the cool plasma associated with TW Hya are indeed redshifted with respect to the stellar photosphere, providing an average receding velocity of $38.3 \pm 5.1 \mathrm{~km} \mathrm{~s}^{-1}$. The cool plasma then has an inward motion in the stellar atmosphere, which is in agreement with predictions based on the accretion shock scenario. This is further strong evidence that the plasma at a few MK on TW Hya, responsible for the soft X-ray emission, is indeed located in the post-shock region and not in accretion-fed coronal loops. This holds not only for the cool plasma as a whole, but also for individual plasma components at low temperatures. We in fact also detected a significant redshift from individual cool lines (see Table A.1 and Fig. 4). In particular, all the cool plasma components at $\sim 2, \sim 3$, and $\sim 4 \mathrm{MK}$ associated with TW Hya, probed by the emission lines of O VII, O VIII, and Ne IX, move with similar inward velocities. On TW Hya, all the cool plasma components responsible for the soft X-ray emission then appear to be located in the post-shock region.

\subsection{Constraints on the accretion geometry from $X$-ray Doppler shift}

We observed that the soft X-ray emission from TW Hya is significantly redshifted, $v_{\mathrm{Xc}}^{\star}=38.3 \pm 5.1 \mathrm{~km} \mathrm{~s}^{-1}$, with respect to the photosphere. The observed radial velocity of the cool plasma on TW Hya allows us to infer constraints on the accretion geometry. The pre-shock accretion velocity is directly probed by the post-shock temperature. The EMD clearly indicates a cool peak at $T \sim 2.5-3 \mathrm{MK}$ (Kastner et al. 2002; Brickhouse et al. 2010). Assuming strong-shock conditions, the predicted postshock temperature $T_{\text {post }}$ depends only on the pre-shock velocity, i.e. $T_{\text {post }}=\left(3 \mu m_{\mathrm{p}} v_{\text {pre }}^{2}\right) /\left(16 k_{\mathrm{B}}\right)$, where $\mu m_{\mathrm{p}}$ is the average particle mass, whose typical value is $\sim 0.6 m_{\mathrm{p}}$ (Argiroffi et al. 2007). Even considering the complex and variable structure of the post-shock plasma, this relation holds (Sacco et al. 2010). Therefore the peak temperature of the EMD suggests an intrinsic pre-shock velocity of $430-470 \mathrm{~km} \mathrm{~s}^{-1}$. This inferred velocity fits neatly between the maximum value provided by the free-fall velocity of $\sim 500 \mathrm{~km} \mathrm{~s}^{-1}$ that characterizes TW Hya, and the minimum, $\sim 300 \mathrm{~km} \mathrm{~s}^{-1}$, needed to have an X-ray emitting post-shock plasma (Sacco et al. 2010).

The X-ray emitting post-shock plasma is expected to show in its X-ray emission a bulk inward velocity of $v_{\text {post }} \approx v_{\text {pre }} / 4 \approx$ $110-120 \mathrm{~km} \mathrm{~s}^{-1}$, even taking into account the complex spatial structure of the post-shock and its intrinsic instabilities (Bonito et al., in prep.). Then, because of the radial velocity of $\sim 40 \mathrm{~km} \mathrm{~s}^{-1}$ that we detected, we can infer an inclination angle between the post-shock velocity direction and line of sight of $\sim 70^{\circ}$. And hence, assuming that the accretion streams arrive perpendicularly with respect to the stellar surface, the base of the accretion streams producing the observed soft X-ray emission from TW Hya appear to be located at low latitudes. Considering that the inclination of TW Hya is low, but not zero, 


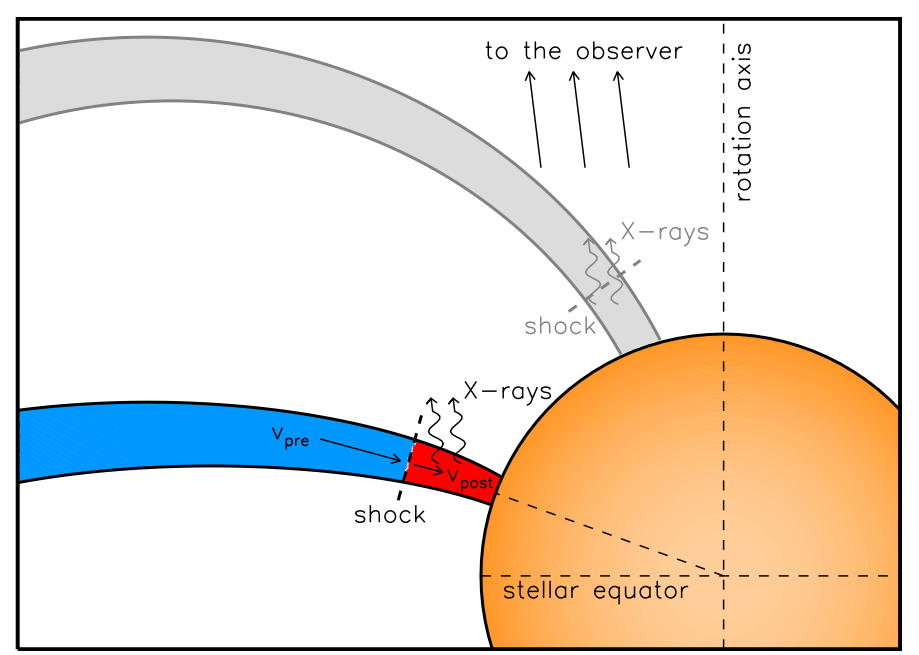

Fig. 6. Schematic illustration of the low-latitude accretion-shock position on the stellar surface of TW Hya, as inferred from the observed X-ray Doppler shift. The red area at the base of the accretion stream indicates the post-shock region, where the high-density plasma at temperature of 2-4 MK, responsible for the emission of cool redshifted X-ray lines, is located. Accretion streams terminating near the stellar pole (as that shown in gray) cannot be excluded, though their X-ray post-shock emission could not in fact be readily observed because of the absorption within the pre-shock material.

and assuming $i=7^{\circ}$ (Qi et al. 2004), we can then infer that the latitude of these X-ray emitting accretion shocks should lie between $10^{\circ}$ and $30^{\circ}$, as shown in Fig. 6. Summarizing, the constraint provided by our analysis is stringent because we have both an inference as to the intrinsic pre-shock velocity, based on the temperature of the X-ray emitting post-shock plasma, and a measurement of the post-shock velocity along the line of sight measured by the redshift. Finally, even guessing that the observed velocity of $\sim 40 \mathrm{~km} \mathrm{~s}^{-1}$ comes out from a mixture of plasma components with radial velocities ranging, for instance, from 0 to $\sim 80 \mathrm{~km} \mathrm{~s}^{-1}$ (because of the spectral resolution of the Chandra/HETGS), the corresponding latitudes would be lower than $50^{\circ}$, confirming also in this case that X-ray emitting accretion spots are mainly located at low latitudes.

The existence of significant accretion terminating at low latitudes confirms that the geometry of the accretion streams depends not only on the dipole component, but on the magnetic configuration near the stellar surface (Gregory et al. 2006) that is characterized on TW Hya by intense higher order magnetic components (Donati et al. 2011).

Because the start and stop times of the TW Hya (1) observation cover a period of $\sim 15 \mathrm{~d}$ and because the redshift appears to be the same on shorter time intervals as well, these low-latitude accretion spots seem to be stable over timescales of a few weeks. Moreover, despite the lower significance level, the same radial velocity also emerges from the TW Hya (2) observation, indicating that the geometry of these low-latitude accretion spots could likely have remained stable over a timescale of a few years. Evidence of long-term stability of the accretion configuration was observed in a few other cases (e.g., Hamaguchi et al. 2012; Sicilia-Aguilar et al. 2015), although accretion more often appears as a highly variable phenomenon (e.g., Sousa et al. 2016; Rigon et al. 2017).

Even if the observed X-ray redshift from TW Hya indicates the existence of an apparently stable low-latitude accretionshock area, X-ray data alone cannot exclude the existence of accretion streams terminating near the stellar pole. X-rays are in fact very easily absorbed by the pre-shock material (Bonito et al. 2014; Costa et al. 2017), hence post-shock X-rays are not expected to be easily observed when a significant portion of preshock material is located along the line of sight (Argiroffi et al. 2011). Because of the almost pole-on view of TW Hya, this could be the case for streams terminating at very high latitudes (Fig. 6).

\subsection{Comparison with other constraints on accretion geometry}

In this work we focused on the X-ray emission due to the post-shock plasma on TW Hya, measuring a redshift of $38.3 \pm$ $5.1 \mathrm{~km} \mathrm{~s}^{-1}$ and inferring that the accretion-shock regions responsible for this emission should be located at low latitude on the stellar surface. In addition to the plasma heated in the accretion shocks, accretion in low-mass stars involves several components, from cold inner disk structures to accretion streams. To try to depict a coherent picture of the accretion process on TW Hya, we compare here our results to those previously obtained exploiting different diagnostics and/or focusing on different accretion components. The C IV line doublets at $1550 \AA$ probes plasma at $\sim 10^{5} \mathrm{~K}$ involved in the accretion process. In CTTS this doublet usually shows a profile characterized by a narrow component (NC) and a broad component (BC). Ardila et al. (2013) suggested that the NC originates in the post-shock region, where the material cools down after having been heated up to few MK, while the BC instead forms in pre-shock portions of the accretion streams. Both the $\mathrm{NC}$ and $\mathrm{BC}$ of the $\mathrm{C}$ IV line profiles of TW Hya are redshifted with peaks located at $\approx 30$ and $\approx 120 \mathrm{~km} \mathrm{~s}^{-1}$, respectively (Ardila et al. 2013). The peak of the $\mathrm{NC}$ is very similar to the velocity we measured for the plasma at $3 \mathrm{MK}$. This strongly suggests that both $\mathrm{C}$ IV NC and X-ray cool lines originate in the same post-shock plasma with the $10^{5} \mathrm{~K}$ material located deeper in the post-shock cooling zone, as predicted by MHD modeling (Colombo et al. 2016). Therefore, the C IV line doublet also points to low-latitude accretion spots on TW Hya.

The absorption cross-section in the soft X-ray band $(\sim 15-20 \AA)$ is similar to that in the far UV band $(\sim 1000-$ $2000 \AA$, Ryter 1996). Therefore, as for the X-rays, UV line photons emitted by the post-shock plasma can easily escape sideways with respect to the stream direction, but are highly absorbed if emitted in the stream direction. Therefore, both UV and soft X-ray lines preferentially probe streams observed in the direction perpendicular to the stream. In the case of TW Hya, therefore, UV emission confirms low-latitude spots, but cannot exclude a polar shock region. Considering in fact the large sample of CTTS inspected by Ardila et al. (2013), the C IV NC shows on average low radial velocities. This could be because this bias is related to the post-shock observability that is related to the stream direction, and not because accretion inflow motions are slower than the free-fall velocity. The MK temperature of the post-shock guarantees, in fact, that the intrinsic pre-shock velocity should be as high as that predicted by free fall, hence the low radial velocity observed on average for the C IV NC (Ardila et al. 2013) indicates that the emitting accretion regions are viewed at high inclination with respect to the stream direction.

A low-latitude accretion spot on TW Hya was previously suggested by Batalha et al. (2002) to interpret veiling and line variations as due to rotational modulation of the hot spot. Moreover the existence of equatorial accretion streams agrees with the magnetic topology of TW Hya, characterized by higher order 
components that dominate the dipolar component (Donati et al. 2011).

As discussed above, the observed X-ray redshift points to a low-latitude stream, but cannot exclude the presence of polar streams. Their existence is indeed suggested by the profile of the He I line at $10380 \AA$. This line clearly shows a subcontinuum redshifted absorption feature extending up to $\sim 300-400 \mathrm{~km} \mathrm{~s}^{-1}$ (Fischer et al. 2008; Dupree et al. 2014). This absorbing material can be obtained only with a free-fall velocity oriented along the line of sight, as is precisely the case for an accretion funnel terminating at high latitude. Donati et al. (2011), performing spectropolarimetric monitoring of the Ca II infrared triplet, and interpreting the line variability as due to rotational modulation, found that both polar and equatorial accretion indeed occur on TW Hya.

Accretion can also be probed by hydrogen Balmer lines. However, interpreting their profiles to infer constraints on the accretion geometry is nontrivial. In TW Hya both $\mathrm{H} \alpha$ and $\mathrm{H} \beta$ lines present a redshifted absorption feature at $\sim 35 \mathrm{~km} \mathrm{~s}^{-1}$ (Alencar \& Batalha 2002; Dupree et al. 2014). This feature appears to be stable in observations performed at different epochs, and it is present also during observations performed simultaneously with the 2007 Chandra observing campaign. This absorption is usually interpreted as due to the hot-spot emission observed through the accretion column, which implies an accretion stream terminating at high latitude (Alencar \& Batalha 2002). However, because of the pole-on inclination of TW Hya, a polar stream would probably produce absorption features at higher velocity. Moreover, this feature provides a redshifted velocity that is similar to that observed from soft X-rays and far UV lines. For this reason, we speculate that models predicting $\mathrm{H} \alpha$ and $\mathrm{H} \beta$ line emission, both from within the outer stellar atmosphere and in post- and pre-shock material, are needed to test whether or not the similar redshifts shown by optical absorption features, far UV lines, and soft X-rays lines, indicate that they all originate from the same structure.

\section{Conclusions}

Thanks to the wavelength resolution and calibration of the Chandra/HETGS gratings, we measured for the first time the redshift, with respect to the stellar photosphere, of the cool X-ray emitting plasma component of the CTTS TW Hya. The observed redshift of $38.3 \pm 5.1 \mathrm{~km} \mathrm{~s}^{-1}$ indicates the following:

- This X-ray emitting plasma at $2-4 \mathrm{MK}$ is receding with respect to the photosphere and is indeed located in the postshock region at the base of the accretion streams, as predicted by several HD and MHD models, strongly confirming the nature of the high-density X-ray emitting plasma component observed in general in CTTS.

- The footpoints of these X-ray emitting accretion streams are located at low latitudes $\left(\sim 10^{\circ}-30^{\circ}\right)$ on the stellar surface, as strictly constrained by comparing the measured redshift with the intrinsic pre-shock velocity required to generate the observed temperature of the post-shock X-ray emitting region.

- The observed velocity is very similar to that of the NC of the $\mathrm{C}$ IV resonance doublet, suggesting that both soft X-rays and far UV lines are emitted by the same low-latitude post-shock regions.

This new detection of the X-ray redshift from material accreting onto a CTTS, in addition to providing constraints on the nature of the X-ray emitting plasma and on the magnetospheric accretion geometry, is also important in light of the future X-ray mission Athena, where the combination of large effective area and high spectral resolution will allow us to extend this diagnostic to a larger and more diverse population of CTTS (Nandra et al. 2013; Barret et al. 2016).

Acknowledgements. We thank the referee, Joel Kastner, for very constructive comments. The scientific results reported in this article are based on observations obtained from the Chandra Data Archive.

\section{References}

Alencar, S. H. P., \& Batalha, C. 2002, ApJ, 571, 378

Anders, E., \& Grevesse, N. 1989, Geochim. Cosmochim. Acta, 53, 197

Andrews, S. M., Wilner, D. J., Zhu, Z., et al. 2016, ApJ, 820, L40

Apai, D., Schneider, G., Grady, C. A., et al. 2015, ApJ, 800, 136

Ardila, D. R., Herczeg, G. J., Gregory, S. G., et al. 2013, ApJS, 207, 1

Argiroffi, C., Maggio, A., \& Peres, G. 2007, A\&A, 465, L5

Argiroffi, C., Maggio, A., Peres, G., et al. 2009, A\&A, 507, 939

Argiroffi, C., Flaccomio, E., Bouvier, J., et al. 2011, A\&A, 530, A1

Argiroffi, C., Maggio, A., Montmerle, T., et al. 2012, ApJ, 752, 100

Armitage, P. J. 2011, ARA\&A, 49, 195

Bailey, III, J. I., White, R. J., Blake, C. H., et al. 2012, ApJ, 749, 16

Baraffe, I., \& Chabrier, G. 2010, A\&A, 521, A44

Baraffe, I., Chabrier, G., \& Gallardo, J. 2009, ApJ, 702, L27

Barret, D., Lam Trong, T., den Herder, J.-W., et al. 2016, in Space Telescopes and Instrumentation 2016: Ultraviolet to Gamma Ray, Proc. SPIE, 9905, 99052F Batalha, C., Batalha, N. M., Alencar, S. H. P., Lopes, D. F., \& Duarte, E. S. 2002, ApJ, 580, 343

Bergin, E. A., Cleeves, L. I., Gorti, U., et al. 2013, Nature, 493, 644

Bonito, R., Orlando, S., Argiroffi, C., et al. 2014, ApJ, 795, L34

Bouvier, J., Forestini, M., \& Allain, S. 1997, A\&A, 326, 1023

Brickhouse, N. S., Dupree, A. K., \& Young, P. R. 2001, ApJ, 562, L75

Brickhouse, N. S., Cranmer, S. R., Dupree, A. K., Luna, G. J. M., \& Wolk, S. 2010, ApJ, 710, 1835

Brickhouse, N. S., Cranmer, S. R., Dupree, A. K., et al. 2012, ApJ, 760, L21

Calvet, N., \& Gullbring, E. 1998, ApJ, 509, 802

Calvet, N., \& Hartmann, L. 1992, ApJ, 386, 239

Chung, S. M., Drake, J. J., Kashyap, V. L., Lin, L. W., \& Ratzlaff, P. W. 2004, ApJ, 606, 1184

Colombo, S., Orlando, S., Peres, G., Argiroffi, C., \& Reale, F. 2016, A\&A, 594, A93

Costa, G., Orlando, S., Peres, G., Argiroffi, C., \& Bonito, R. 2017, A\&A, 597, A1

Curran, R. L., Argiroffi, C., Sacco, G. G., et al. 2011, A\&A, 526, A104

Debes, J. H., Jang-Condell, H., Weinberger, A. J., Roberge, A., \& Schneider, G. 2013, ApJ, 771, 45

Debes, J. H., Poteet, C. A., Jang-Condell, H., et al. 2017, ApJ, 835, 205

Donaldson, J. K., Weinberger, A. J., Gagné, J., et al. 2016, ApJ, 833, 95

Donati, J.-F., \& Landstreet, J. D. 2009, ARA\&A, 47, 333

Donati, J.-F., Gregory, S. G., Alencar, S. H. P., et al. 2011, MNRAS, 417, 472

Drake, J. J. 2005, in 13th Cambridge Workshop on Cool Stars, Stellar Systems and the Sun, eds. F. Favata, G. A. J. Hussain, \& B. Battrick, ESA SP, 560, 519 Drake, J. J., Testa, P., \& Hartmann, L. 2005, ApJ, 627, L149

Drake, J. J., Ratzlaff, P. W., Laming, J. M., \& Raymond, J. 2009, ApJ, 703, 1224 Dupree, A. K., Brickhouse, N. S., Cranmer, S. R., et al. 2014, ApJ, 789, 27 Ercolano, B., Rosotti, G. P., Picogna, G., \& Testi, L. 2017, MNRAS, 464, L95 Fischer, W., Kwan, J., Edwards, S., \& Hillenbrand, L. 2008, ApJ, 687, 1117 Gaia Collaboration (Brown, A. G. A., et al.) 2016, A\&A, 595, A2

Gontcharov, G. A. 2006, Astron. Lett., 32, 759

Gregory, S. G., Jardine, M., Simpson, I., \& Donati, J.-F. 2006, MNRAS, 371, 999

Gregory, S. G., Matt, S. P., Donati, J.-F., \& Jardine, M. 2008, MNRAS, 389, 1839

Guarcello, M. G., Flaccomio, E., Micela, G., et al. 2017, A\&A, 602, A10

Güdel, M., \& Telleschi, A. 2007, A\&A, 474, L25

Güdel, M., Skinner, S. L., Mel'Nikov, S. Y., et al. 2007, A\&A, 468, 529

Gullbring, E. 1994, A\&A, 287, 131

Günther, H. M., Liefke, C., Schmitt, J. H. M. M., Robrade, J., \& Ness, J.-U. 2006, A\&A, 459, L29

Hamaguchi, K., Grosso, N., Kastner, J. H., et al. 2012, ApJ, 754, 32

Hartmann, L., Hewett, R., \& Calvet, N. 1994, ApJ, 426, 669

Hartmann, L., Herczeg, G., \& Calvet, N. 2016, ARA\&A, 54, 135

Hashimoto, J., Tamura, M., Muto, T., et al. 2011, ApJ, 729, L17

Herbst, W., \& Koret, D. L. 1988, AJ, 96, 1949 
Herczeg, G. J., \& Hillenbrand, L. A. 2014, ApJ, 786, 97

Huélamo, N., Figueira, P., Bonfils, X., et al. 2008, A\&A, 489, L9

Huenemoerder, D. P., Kastner, J. H., Testa, P., Schulz, N. S., \& Weintraub, D. A 2007, ApJ, 671, 592

Huenemoerder, D. P., Mitschang, A., Dewey, D., et al. 2011, AJ, 141, 129

Hughes, A. M., Wilner, D. J., Calvet, N., et al. 2007, ApJ, 664, 536

Ingleby, L., Calvet, N., Herczeg, G., et al. 2013, ApJ, 767, 112

Ishibashi, K., Dewey, D., Huenemoerder, D. P., \& Testa, P. 2006, ApJ, 644, L117

Johns-Krull, C. M. 2007, ApJ, 664, 975

Johns-Krull, C. M., \& Valenti, J. A. 2001, in Young Stars Near Earth: Progress and Prospects, eds. R. Jayawardhana, \& T. Greene, ASP Conf. Ser., 244, 147

Kastner, J. H., Zuckerman, B., Weintraub, D. A., \& Forveille, T. 1997, Science, 277, 67

Kastner, J. H., Huenemoerder, D. P., Schulz, N. S., Canizares, C. R., \& Weintraub, D. A. 2002, ApJ, 567, 434

Königl, A. 1991, ApJ, 370, L39

Lawson, W. A., \& Crause, L. A. 2005, MNRAS, 357, 1399

Massarotti, A., Latham, D. W., Stefanik, R. P., \& Fogel, J. 2008, AJ, 135, 209

Matsakos, T., Chièze, J.-P., Stehlé, C., et al. 2013, A\&A, 557, A69

Muzerolle, J., Calvet, N., Briceño, C., Hartmann, L., \& Hillenbrand, L. 2000, ApJ, 535, L47

Nandra, K., Barret, D., Barcons, X., et al. 2013, ArXiv e-prints [arXiv: 1306.2307$]$

Ness, J.-U., \& Schmitt, J. H. M. M. 2005, A\&A, 444, L41

Nidever, D. L., Marcy, G. W., Butler, R. P., Fischer, D. A., \& Vogt, S. S. 2002, ApJS, 141, 503

Nordström, B., Mayor, M., Andersen, J., et al. 2004, A\&A, 418, 989

Orlando, S., Sacco, G. G., Argiroffi, C., et al. 2010, A\&A, 510, A71

Orlando, S., Bonito, R., Argiroffi, C., et al. 2013, A\&A, 559, A127
Qi, C., Ho, P. T. P., Wilner, D. J., et al. 2004, ApJ, 616, L11

Rigon, L., Scholz, A., Anderson, D., \& West, R. 2017, MNRAS, 465, 3889

Romanova, M. M., \& Owocki, S. P. 2015, Space Sci. Rev., 191, 339

Romanova, M. M., Ustyugova, G. V., Koldoba, A. V., Wick, J. V., \& Lovelace, R. V. E. 2003, ApJ, 595, 1009

Romanova, M. M., Ustyugova, G. V., Koldoba, A. V., \& Lovelace, R. V. E. 2004, ApJ, 610, 920

Romanova, M. M., Long, M., Lamb, F. K., Kulkarni, A. K., \& Donati, J.-F. 2011, MNRAS, 411, 915

Ryter, C. E. 1996, Ap\&SS, 236, 285

Sacco, G. G., Argiroffi, C., Orlando, S., et al. 2008, A\&A, 491, L17

Sacco, G. G., Orlando, S., Argiroffi, C., et al. 2010, A\&A, 522, A55

Schmitt, J. H. M. M., Robrade, J., Ness, J.-U., Favata, F., \& Stelzer, B. 2005, A\&A, 432, L35

Schneider, P. C., Günther, H. M., \& Robrade, J. 2017, Astron. Nachr., 338, 201

Setiawan, J., Henning, T., Launhardt, R., et al. 2008, Nature, 451, 38

Sicilia-Aguilar, A., Fang, M., Roccatagliata, V., et al. 2015, A\&A, 580, A82

Siwak, M., Rucinski, S. M., Matthews, J. M., et al. 2011, MNRAS, 410, 2725

Siwak, M., Rucinski, S. M., Matthews, J. M., et al. 2014, MNRAS, 444, 327

Smith, R. K., Brickhouse, N. S., Liedahl, D. A., \& Raymond, J. C. 2001, ApJ, 556, L91

Sousa, A. P., Alencar, S. H. P., Bouvier, J., et al. 2016, A\&A, 586, A47

Stelzer, B., \& Schmitt, J. H. M. M. 2004, A\&A, 418, 687

Ulrich, R. K. 1976, ApJ, 210, 377

Vacca, W. D., \& Sandell, G. 2011, ApJ, 732, 8

Webb, R. A., Zuckerman, B., Platais, I., et al. 1999, ApJ, 512, L63

Williams, J. P., \& Cieza, L. A. 2011, ARA\&A, 49, 67

Zel'dovich, Y. B., \& Raizer, Y. P. 1967, Physics of shock waves and hightemperature hydrodynamic phenomena (New York: Academic Press) 


\section{Appendix A: Line Doppler shifts}

We show in Figs. A.1 and A.2 the observed and fitted line profiles, for both HEG and MEG gratings, for all the inspected observations. The velocities corresponding to the displacement of each line with respect to its predicted rest wavelength are listed in Table A.1. We also report in Table A.1 the cool and hot plasma velocities of each observation, obtained by computing the weighted average of the velocities of cool and hot line subsamples, as classified in Table 3. 
C. Argiroffi et al.: Redshifted X-rays from TW Hya: A low-latitude accretion spot

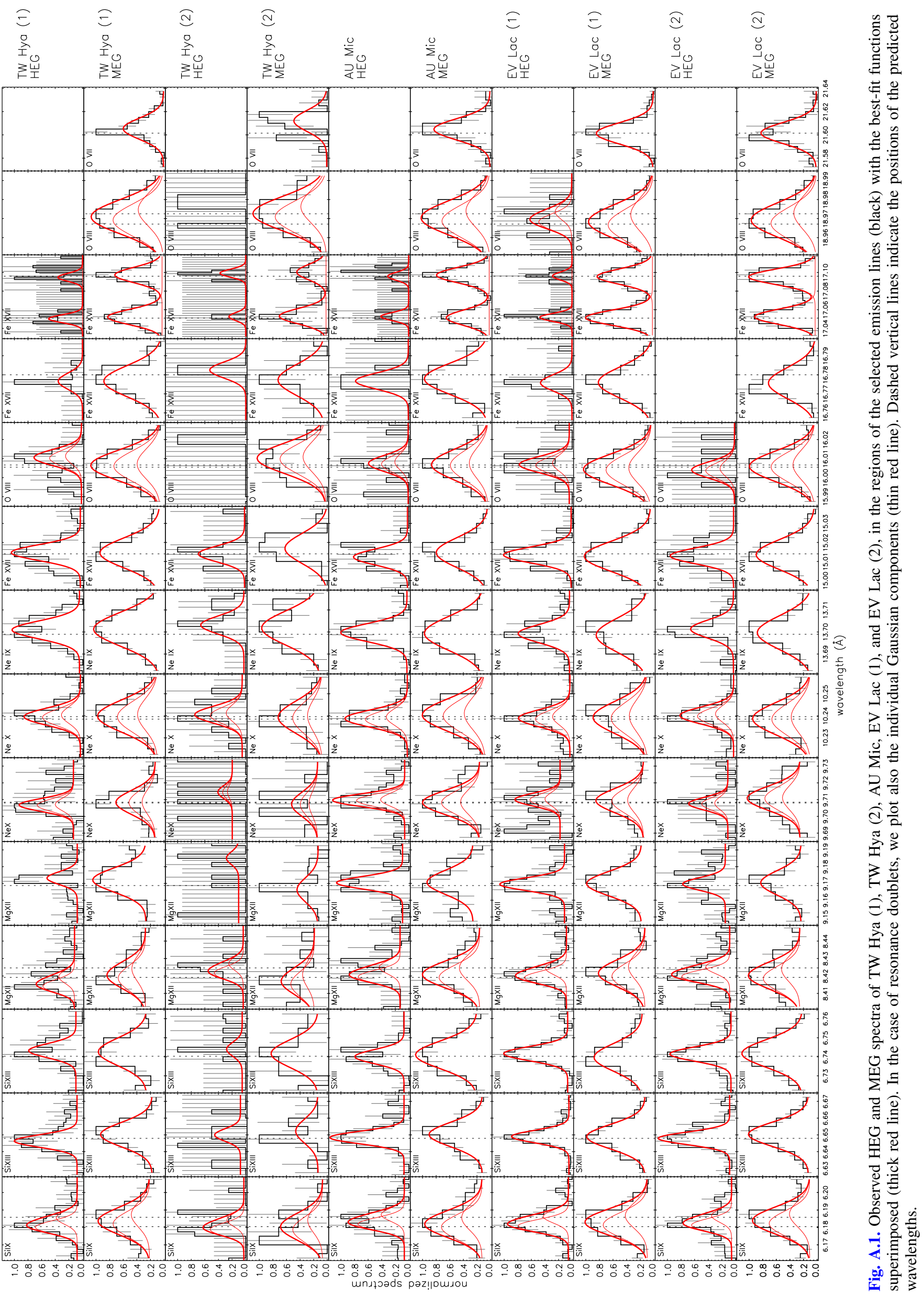




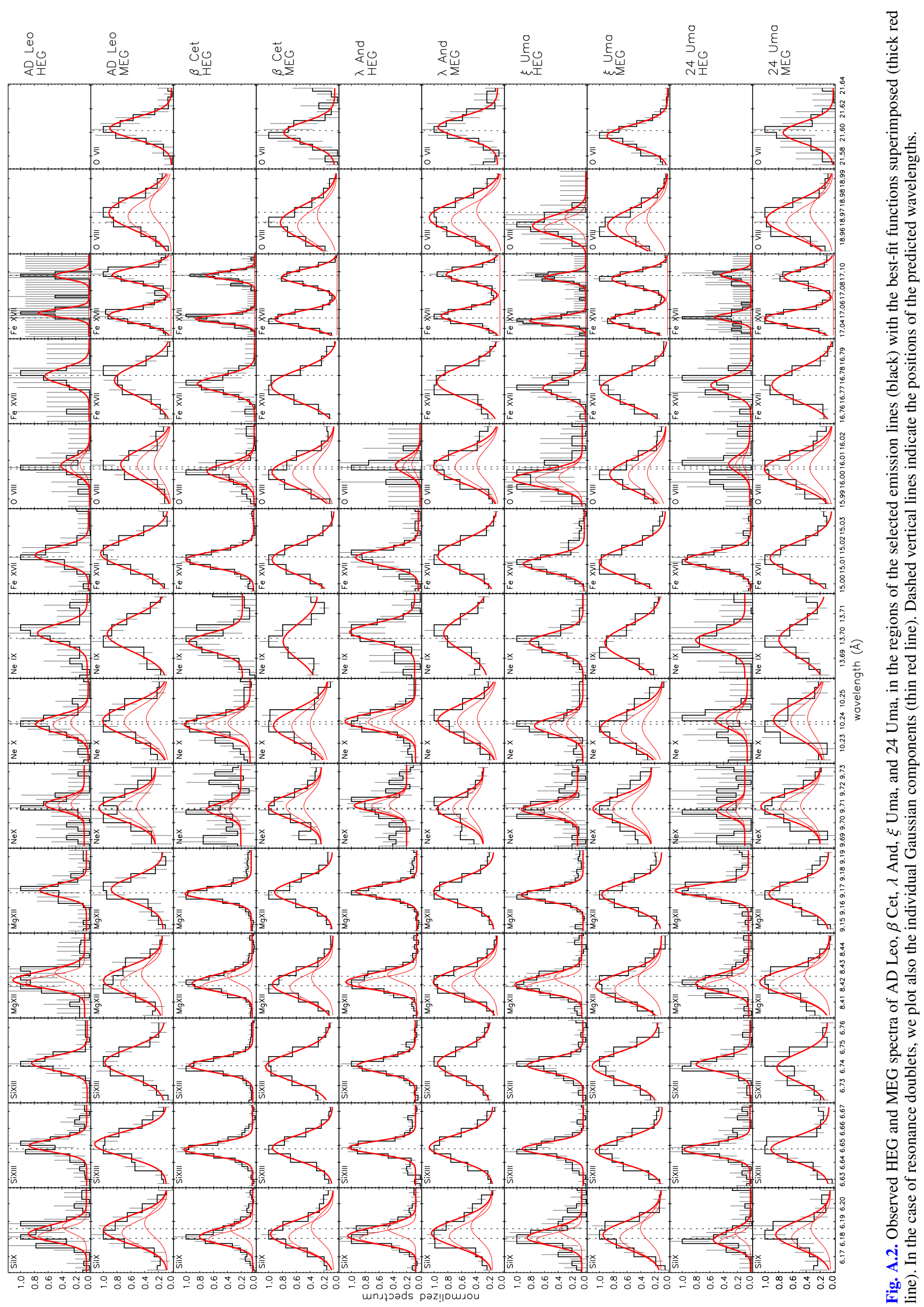




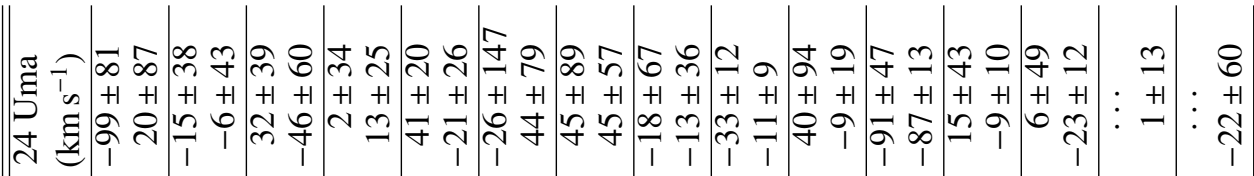

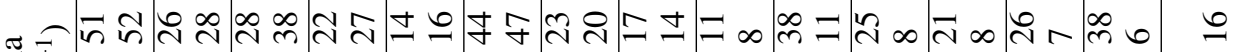
每 $+1+1+1+1+1+1+1+1+1+1+1+1+1+1+1+1+1+1+1+1+1+1+1+1+1+1+1+1$. +1

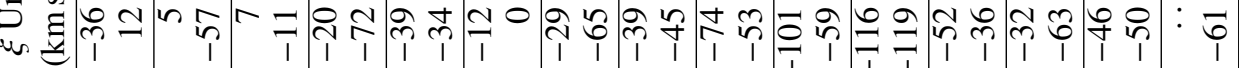

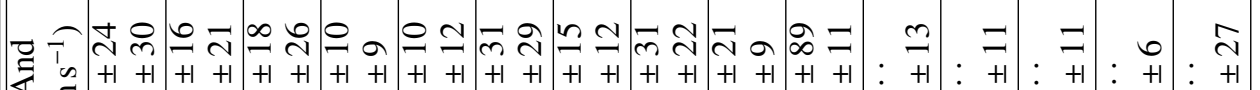

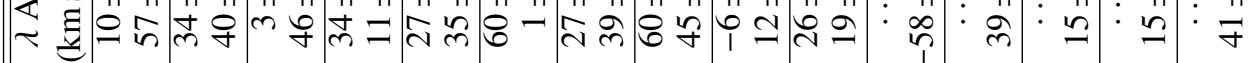

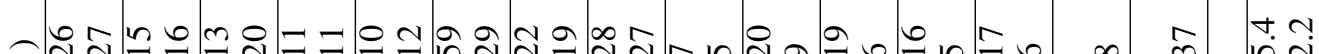

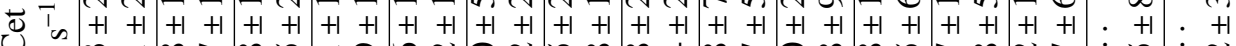

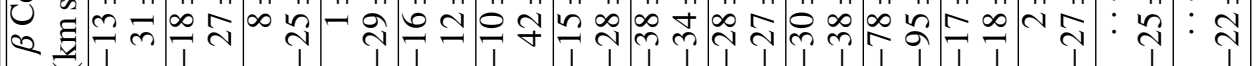

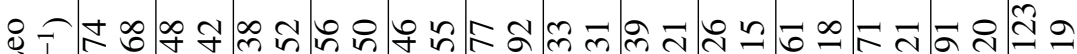

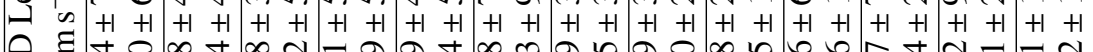

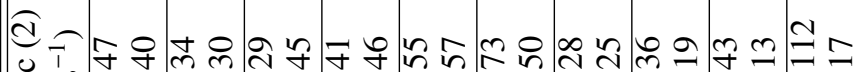

$\pi^{2}=0$

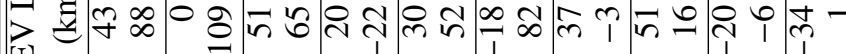

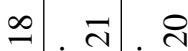

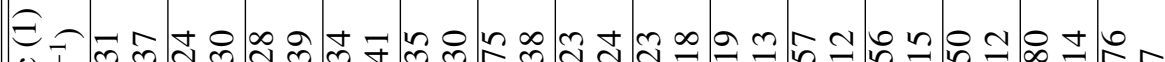

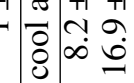
$\approx 0=1+1+1+1+1+1+1+1+1+1+1+1+1+1+1+1+1+1+1+1+1+1+1+1+1+1+1:$

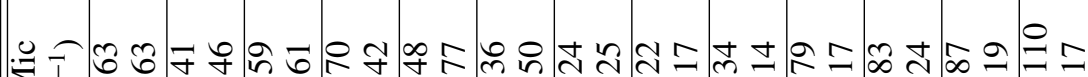

$\sum_{\infty}+1+1+1+1+1+1+1+1+1+1+1+1+1+1+1+1+1+1+1+1+1+1+1+1+1+1$

不

产

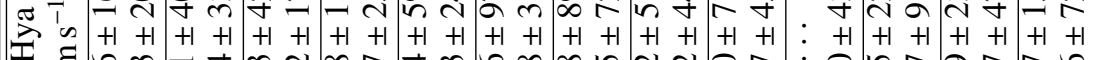

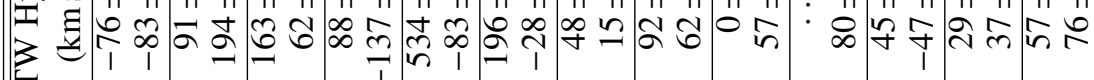

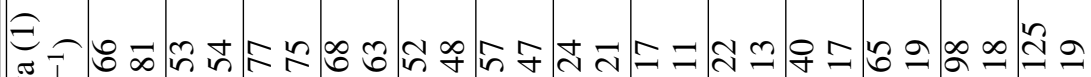

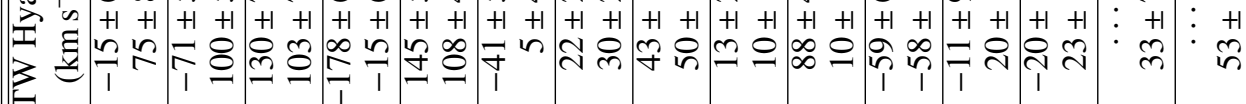

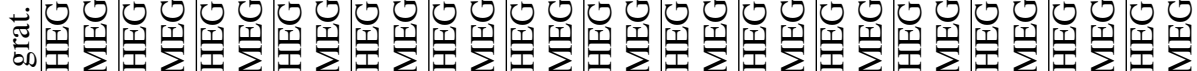

\begin{tabular}{|c|c|c|c|c|c|c|c|c|c|c|c|c|c|}
\hline $\begin{array}{l}\stackrel{+}{D} \\
\underset{6}{0}\end{array}$ & $\begin{array}{l}2 \\
\text { ț } \\
\text { : }\end{array}$ & $\frac{\hat{o}}{\stackrel{+}{0}}$ & 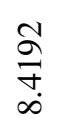 & $\begin{array}{l}\infty \\
\infty \\
a \\
a\end{array}$ & 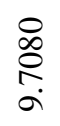 & $\begin{array}{l}\mathscr{\infty} \\
\infty \\
\\
ٍ \\
0\end{array}$ & $\begin{array}{l}\stackrel{2}{\circ} \\
\text { ఏ) } \\
\end{array}$ & $\begin{array}{l}\stackrel{q}{+} \\
\text { o } \\
\stackrel{n}{n}\end{array}$ & $\begin{array}{l}n \\
\tilde{8} \\
0 \\
0\end{array}$ & $\begin{array}{l}8 \\
8 \\
0 \\
6 \\
0\end{array}$ & $\begin{array}{l}0 \\
n \\
0 \\
\end{array}$ & $\begin{array}{l}8 \\
8 \\
8 \\
1 \\
=\end{array}$ & $\begin{array}{l}\overrightarrow{\widehat{\sigma}} \\
\infty \\
\infty \\
\infty\end{array}$ \\
\hline$\underset{.}{\Delta}$ & $\underset{.}{\mid Z}$ & $\underset{:}{\mathbb{Z}}$ & $\begin{array}{l}\overline{\bar{x}} \\
\sum^{\infty}\end{array}$ & $\begin{array}{l}\bar{x} \\
\sum^{\infty}\end{array}$ & $\begin{array}{l}x \\
0 \\
j\end{array}$ & $\begin{array}{l}\grave{u} \\
\stackrel{0}{z}\end{array}$ & $\frac{u}{u}$ & $\begin{array}{l}\underset{x}{x} \\
0 \\
0 \\
0\end{array}$ & $\stackrel{\Xi}{z}$ & $\begin{array}{l}\vec{x} \\
x \\
0 \\
0\end{array}$ & $\begin{array}{l}\sum_{x} \\
0 \\
0\end{array}$ & $\begin{array}{l}\sum_{x} \\
0 \\
\omega \\
L\end{array}$ & F \\
\hline
\end{tabular}

